\title{
Design for structural vibration suppression in laminate acoustic metamaterials
}

\author{
$\mathrm{ZC} \mathrm{He}^{1}, \mathrm{X} \mathrm{Xiao}^{1}, \mathrm{Eric} \mathrm{Li}^{1,2 *}$, \\ ${ }^{I}$ State Key Laboratory of Advanced Design and Manufacturing for Vehicle Body, Hunan University, Changsha, \\ 410082 P. R. China \\ ${ }^{2}$ Department of Mechanical and Automation Engineering, The Chinese University of Hong Kong, Shatin, NT, \\ Hong Kong, China
}

\begin{abstract}
This paper investigates the stopband of laminate acoustic metamaterials, which is composed of carbon-fiber-reinforced polymer (CFRP) and a periodic array of mass-spring-damper subsystems integrated with the laminates to act as vibration absorbers. Based on the mathematical model derived in this work, a wide stopband is observed by dispersion analysis. The frequency response analysis is performed to confirm its stopband behavior for a finite laminate acoustic metamaterial. Due to the superior strength to weight ratio of CFRP, the laminate acoustic metamaterials are able to have a much wider stopband than the conventional metamaterial plates proposed in recent years. In addition, the effects of the relevant parameters on the stopband of laminate acoustic metamaterial are discussed in this work. The excellent performance of laminate acoustic metamaterials has been applied to design the vehicle door, and the vibration of the vehicle door is suppressed significantly.
\end{abstract}

Key words: laminate acoustic metamaterials; stop-band; local resonance; CFRP

*Corresponding author: Eric Li

Email address: ericsg2012@gmail.com 


\section{Introduction}

As early as over fifty years ago, the electromagnetic metamaterials were first proposed with its unusual properties that do not exist in nature ${ }^{[1-3]}$, such as negative permittivity and permeability. The electromagnetic metamaterials are mainly designed by using the effects of negative refractive indices, cloaking and superlensing. Over the last few years ${ }^{[4-7]}$, acoustic metamaterials (AMs) with negative effective density and stiffness have received increasing attention. The concept of AMs extends far beyond of negative refraction in EMs, rather giving vast choice of material parameters for different applications. Currently AMs are widely applied in many fields, including national defense fields for acoustic collimating ${ }^{[10]}$ and sound insulation fields for elastic wave absorption ${ }^{[11,12,21,22]}$.

The concept of acoustic metamaterials was developed by phononic crystals (PCs). Analogous to the photonic crystals, the stopband or bandgaps within which the propagation of elastic or acoustic waves are inhibited exist in the PCs ${ }^{[22,32-34]}$. The earlier studies have shown that there are two working mechanisms of stopband in PCs, which are known as Bragg scattering and local resonance. For Bragg scattering PCs, the low-frequency vibration suppression depends much on the size of PCs ${ }^{[11]}$. To solve this problem, Liu et al ${ }^{[13]}$ proposed the first acoustic metamaterials based on the local resonance. This type of AMs is also known as locally resonant sonic materials (LRSMs), and the stopband can be obtained two orders of magnitude lower than that of the Bragg gaps. The AMs have successfully achieved a small size structure to control the large wavelength (low frequency) sonic waves. In general, the AMs are composed of periodically arranged low-frequency resonators consisting of a solid core material with relatively high density coated by an elastically soft material. Wang ${ }^{[14]}$ proposed one-dimensional phononic 
crystals with locally resonant structures comprehensively. $\mathrm{Yu}^{[15]}$ studied flexural vibration band gaps in a Timoshenko beam with locally resonant structures. Similar works by Phani ${ }^{[18]}$ show that the stopband also exists in two-dimensional metamaterial structures with periodic local resonators. In 2007, the characteristics of wave propagation during pass bands in twodimensional thin plate phononic crystals were analyzed ${ }^{[16]}$. Furthermore, metamaterials with simultaneously negative bulk modulus and mass density were investigated by Ding et al. ${ }^{[17,19]}$.

However, the negative effective mass and modulus only exist in a narrow frequency range for most of the acoustic metamaterials. Therefore, the design of structures with broadband negative parameters is an important issue in the field of acoustic metamaterials. Recently, Peng ${ }^{[20]}$ proposed the metamaterial plate with multi-frequency vibration absorbers for broad-band elastic wave absorption, which is based on the idea of local resonance. The metamaterial plate composed of two-degree of freedom (DOF) subsystems generates two stopbands, and the stopband can be widened by reducing the mass of plate or increasing the mass of vibration absorbers. Nevertheless, a big mass of vibration absorbers may not be very effective in the practical application.

Hence, the laminate metamaterials composed of composite materials such as carbon-fiberreinforced polymer (CFRP), which has the better strength to weight ratio compared with traditional homogeneous materials like Steel or Aluminum, may be a better choice. The material property of the composites can be engineered according to the application requirements ${ }^{[26-29,35-40]}$. Applications like aerospace components ${ }^{[30]}$, where the weight is a decisive factor, can benefit tremendously with the usage of composite materials. However, the undesirable behaviors such as extensional-shear coupling and bending-twist coupling ${ }^{[31]}$, which greatly increase the 
complexity of the design task, are encountered with composite materials. In order to avoid these undesirable behaviors in engineering, the symmetric laminate may be the best option.

To the best of our knowledge, laminate acoustic metamaterials that consist of orthotropic laminates and mass-spring-damper subsystems have never been presented in the literature. This paper aims to design composite laminate acoustic metamaterials for broadband vibration absorption with the superior strength to weight ratio of CFRP. The paper is organized as follows. Firstly, in Section 2, the basic theory of laminate plate is presented in detail. The behavior of stopband of laminate acoustic metamaterial is analyzed using dispersion analysis in Section 3. Subsequently the validation of the stopband of laminate acoustic metamaterials is described by frequency response analysis. In addition, the working mechanism of the bandgaps is revealed on the basis of the concept of conventional vibration absorbers. The influences of the absorbers' resonant frequencies, boundary conditions of the vibration absorbers on laminates and damping ratios of the vibration absorbers as well as the laminate's mode shapes are investigated. In Section 4, we design the vehicle door composed of low-frequency symmetric laminate acoustic metamaterials with a wide stopband by selecting appropriate masses and springs for subsystems and properly locating them on the laminate acoustic metamaterials. Finally, the results as well as the conclusions of the paper are summarized.

\section{Basic theory of laminate plate}

According to the Kirchhoff Plate theory, the displacement of the plane at any point can be approximated by the following equation

$$
u=u^{0}-z \frac{\partial w}{\partial x} \quad v=v^{0}-z \frac{\partial w}{\partial y} \quad w=w
$$


where $u^{0}$ is the mid-plane displacement in the $x$-axis, and $v^{0}$ is the mid-plane displacement in the $y$-axis.

The relationship of strain-displacement for Kirchhoff Plate is derived based on the linear elastic 2D strain-displacement relationship.

$$
\varepsilon_{x}=\frac{\partial u^{0}}{\partial x}-z \frac{\partial^{2} w}{\partial x^{2}} \quad \varepsilon_{y}=\frac{\partial v^{0}}{\partial y}-z \frac{\partial^{2} w}{\partial y^{2}} \quad \gamma_{x y}=\frac{\partial u^{0}}{\partial y}+\frac{\partial v^{0}}{\partial x}-2 z \frac{\partial^{2} w}{\partial x \partial y}
$$

Eq. (2) can be written in the matrix form:

$$
\begin{gathered}
\left\{\begin{array}{l}
\varepsilon_{x} \\
\varepsilon_{y} \\
\gamma_{x y}
\end{array}\right\}=\left\{\begin{array}{l}
\varepsilon_{x}^{0} \\
\varepsilon_{y}^{0} \\
\gamma_{x y}^{0}
\end{array}\right\}+z\left\{\begin{array}{l}
k_{x} \\
k_{y} \\
k_{x y}
\end{array}\right\} \\
\{\boldsymbol{\varepsilon}\}=\left\{\boldsymbol{\varepsilon}^{0}\right\}+z\{\mathbf{k}\}
\end{gathered}
$$

where $\left\{\boldsymbol{\varepsilon}^{0}\right\}$ is the mid-plane strain vector, $\{\mathbf{k}\}$ is the mid-plane curvature variation vector.

In terms of engineering constants, the orthotropic compliance matrix is defined as:

$$
\left(\begin{array}{l}
\varepsilon_{1} \\
\varepsilon_{2} \\
\varepsilon_{3} \\
\gamma_{12} \\
\gamma_{23} \\
\gamma_{13}
\end{array}\right)=\left[\begin{array}{cccccc}
\frac{1}{E_{1}} & \frac{-v_{21}}{E_{2}} & \frac{-v_{31}}{E_{3}} & 0 & 0 & 0 \\
\frac{-v_{12}}{E_{1}} & \frac{1}{E_{2}} & \frac{-v_{32}}{E_{3}} & 0 & 0 & 0 \\
\frac{-v_{13}}{E_{1}} & \frac{-v_{23}}{E_{2}} & \frac{1}{E_{3}} & 0 & 0 & 0 \\
0 & 0 & 0 & \frac{1}{G_{12}} & 0 & 0 \\
0 & 0 & 0 & 0 & \frac{1}{G_{23}} & 0 \\
0 & 0 & 0 & 0 & 0 & \frac{1}{G_{13}}
\end{array}\right]\left(\begin{array}{c}
\sigma_{1} \\
\sigma_{2} \\
\sigma_{3} \\
\tau_{12} \\
\tau_{23} \\
\tau_{13}
\end{array}\right)
$$


The strain-stress relationship for 2D orthotropic plane stress problems is written as follows:

$$
\begin{gathered}
\sigma_{3}=\tau_{13}=\tau_{23}=0 \\
\left(\begin{array}{l}
\varepsilon_{1} \\
\varepsilon_{2} \\
\gamma_{12}
\end{array}\right)=\left[\begin{array}{ccc}
\frac{1}{E_{1}} & \frac{-v_{12}}{E_{1}} & 0 \\
\frac{-v_{12}}{E_{1}} & \frac{1}{E_{2}} & 0 \\
0 & 0 & \frac{1}{G_{12}}
\end{array}\right]\left(\begin{array}{l}
\sigma_{1} \\
\sigma_{2} \\
\tau_{12}
\end{array}\right]=\left[\begin{array}{ccc}
S_{11} & S_{12} & 0 \\
S_{12} & S_{22} & 0 \\
0 & 0 & S_{44}
\end{array}\right]\left\{\begin{array}{l}
\sigma_{1} \\
\sigma_{2} \\
\tau_{12}
\end{array}\right\}
\end{gathered}
$$

with $\varepsilon_{3}=-\left(\frac{v_{12}}{E_{1}} \sigma_{1}+\frac{v_{23}}{E_{2}} \sigma_{2}\right)$

On the other hand, Eq. (7) can be defined as:

$$
\left\{\begin{array}{l}
\sigma_{1} \\
\sigma_{2} \\
\tau_{12}
\end{array}\right\}=\left[\begin{array}{ccc}
Q_{11} & Q_{12} & 0 \\
Q_{12} & Q_{22} & 0 \\
0 & 0 & Q_{44}
\end{array}\right]\left\{\begin{array}{c}
\varepsilon_{1} \\
\varepsilon_{2} \\
\gamma_{12}
\end{array}\right\}
$$

where:

$$
\begin{array}{ll}
Q_{11}=\frac{S_{22}}{S_{11} S_{22}-S_{12} S_{12}}=\frac{E_{1}}{1-v_{12} v_{21}} & Q_{12}=\frac{-S_{12}}{S_{11} S_{22}-S_{12} S_{12}}=\frac{v_{12} E_{2}}{1-v_{12} v_{21}} \\
Q_{22}=\frac{S_{11}}{S_{11} S_{22}-S_{12} S_{12}}=\frac{E_{2}}{1-v_{12} v_{21}} & Q_{44}=\frac{1}{S_{44}}=G_{12}
\end{array}
$$

The constitutive stress/strain relationships developed so far are all written in the principal material 1-, 2-, 3- coordinate system as given by Eq. (8). However, in order to determine the global behavior of a ply, we need to write this relationship in the global $x$-, $y$-, $z$ - coordinate system. This is accomplished by using the $2 D$ plane stress transformations, as shown below: 


$$
\left\{\boldsymbol{\sigma}_{x}\right\}=[\mathbf{T S}]^{-1}\left\{\boldsymbol{\sigma}_{1}\right\}=[\mathbf{T S}]^{-1}[\mathbf{Q}]\left\{\boldsymbol{\varepsilon}_{1}\right\}=[\mathbf{T S}]^{-1}[\mathbf{Q}][\mathbf{T S}]^{-\mathrm{T}}\left\{\boldsymbol{\varepsilon}_{x}\right\}=[\overline{\mathbf{Q}}]\left\{\boldsymbol{\varepsilon}_{x}\right\}
$$

where $[\overline{\mathbf{Q}}]$ is stiffness matrix on global coordinate.

As shown in Fig. 1, the transformation matrix $2 D$ tensors can be written:

$$
\begin{gathered}
{[\mathbf{T S}]^{-1}=\left[\begin{array}{ccc}
\cos ^{2} \theta & \sin ^{2} \theta & -2 \cos \theta \sin \theta \\
\sin ^{2} \theta & \cos ^{2} \theta & 2 \cos \theta \sin \theta \\
\cos \theta \sin \theta & -\cos \theta \sin \theta & \cos ^{2} \theta-\sin ^{2} \theta
\end{array}\right]} \\
{[\mathbf{T S}]^{-\mathrm{T}}=\left[\begin{array}{ccc}
\cos ^{2} \theta & \sin ^{2} \theta & \cos \theta \sin \theta \\
\sin ^{2} \theta & \cos ^{2} \theta & -\cos \theta \sin \theta \\
-2 \cos \theta \sin \theta & 2 \cos \theta \sin \theta & \cos ^{2} \theta-\sin ^{2} \theta
\end{array}\right]}
\end{gathered}
$$

Substitute Eqs. (10) and (11) into Eq. (9).

$$
\begin{aligned}
& \bar{Q}_{11}=\cos ^{4} \theta Q_{11}+\sin ^{4} \theta Q_{22}+2 \cos ^{2} \theta \sin ^{2} \theta\left(Q_{12}+2 Q_{44}\right) \\
& \bar{Q}_{22}=\sin ^{4} \theta Q_{11}+\cos ^{4} \theta Q_{22}+2 \cos ^{2} \theta \sin ^{2} \theta\left(Q_{12}+2 Q_{44}\right) \\
& \bar{Q}_{44}=\left(Q_{11}+Q_{22}-2 Q_{12}\right) \cos ^{2} \theta \sin ^{2} \theta+\left(\cos ^{2} \theta-\sin ^{2} \theta\right) Q_{44} \\
& \bar{Q}_{12}=\bar{Q}_{21}=\left(\cos ^{4} \theta+\sin ^{4} \theta\right) Q_{12}+\left(Q_{11}+Q_{22}-4 Q_{44}\right) \cos ^{2} \theta \sin ^{2} \theta \\
& \bar{Q}_{14}=\bar{Q}_{41}=\left[\cos ^{2} \theta\left(Q_{11}-Q_{12}-2 Q_{44}\right)+\sin ^{2} \theta\left(Q_{12}-Q_{22}+2 Q_{44}\right)\right] \cos \theta \sin \theta \\
& \bar{Q}_{24}=\bar{Q}_{42}=\left[\cos ^{2} \theta\left(Q_{12}-Q_{22}+2 Q_{44}\right)+\sin ^{2} \theta\left(Q_{11}-Q_{12}-2 Q_{44}\right)\right] \cos \theta \sin \theta
\end{aligned}
$$

The positive sign convention for homogeneous or laminated plate mid-plane forces is given in Fig. 2(a). For a homogenous single ply plate of constant thickness, the mid-plane forces can be written in terms of stress variation through the thickness of the plate $(t)$ :

$$
\left\{\begin{array}{l}
N_{x} \\
N_{y} \\
N_{x y}
\end{array}\right\}=\int_{-t / 2}^{t / 2}\left\{\begin{array}{l}
\sigma_{x} \\
\sigma_{y} \\
\tau_{x y}
\end{array}\right\} d z
$$


For a laminated plate made up of ' $n$ ' constant thickness plies, the mid-plane forces can be written in terms of the sum of the stress variation through the thickness of each ply:

$$
\left\{\begin{array}{l}
N_{x} \\
N_{y} \\
N_{x y}
\end{array}\right\}=\sum_{k=1}^{n} \int_{z_{k-1}}^{z_{k}}\left\{\begin{array}{l}
\sigma_{x} \\
\sigma_{y} \\
\tau_{x y}
\end{array}\right\} d z
$$

The positive sign convention of mid-plane moments is given in Fig. 2(b) for homogeneous or laminated plate. Similarly, the mid-plane moments can be expressed in terms of stress variation through the thickness of the plate:

$$
\left\{\begin{array}{l}
M_{x} \\
M_{y} \\
M_{x y}
\end{array}\right\}=\int_{-t / 2}^{t / 2} z\left\{\begin{array}{l}
\sigma_{x} \\
\sigma_{y} \\
\tau_{x y}
\end{array}\right\} d z
$$

For a laminated plate made up of ' $n$ ' constant thickness piles, the mid-plane moments can be expressed in the following form:

$$
\left\{\begin{array}{l}
M_{x} \\
M_{y} \\
M_{x y}
\end{array}\right\}=\sum_{k=1}^{n} \int_{z_{k-1}}^{z_{k}} z\left\{\begin{array}{l}
\sigma_{x} \\
\sigma_{y} \\
\tau_{x y}
\end{array}\right\} d z
$$

Using the Eq. (9), we can apply the equation on the laminated coordinates by adding the subscript ' $k$ ' for each ply:

$$
\left\{\boldsymbol{\sigma}_{x}\right\}_{k}=[\overline{\mathbf{Q}}]_{k}\left\{\boldsymbol{\varepsilon}_{x}\right\}_{k}
$$

Substitute Eqs. (4) and (17) into Eq. (14), the mid-plane forces are written as follows:

$$
\left\{\mathbf{N}_{x}\right\}=\sum_{k=1}^{n} \int_{z_{k-1}}^{z_{k}}\left([\overline{\mathbf{Q}}]_{k}\left(\left\{\boldsymbol{\varepsilon}_{x}^{0}\right\}_{k}+z_{k}\{\mathbf{k}\}_{k}\right)\right) d z
$$

Eq. (18) can be also expressed in the following form: 


$$
\left\{\mathbf{N}_{x}\right\}=[\mathbf{A}]\left\{\boldsymbol{\varepsilon}_{x}^{0}\right\}+[\mathbf{B}]\left\{\mathbf{k}_{x}\right\}
$$

where:

$$
\begin{aligned}
& {[\mathbf{A}]=\sum_{k=1}^{n}\left([\overline{\mathbf{Q}}]_{k}\left(z_{k}-z_{k-1}\right)\right)} \\
& {[\mathbf{B}]=\frac{1}{2} \sum_{k=1}^{n}\left([\overline{\mathbf{Q}}]_{k}\left(z_{k}^{2}-z_{k-1}^{2}\right)\right)}
\end{aligned}
$$

Similarly, the mid-plane moments can be derived as follows:

$$
\left\{\mathbf{M}_{x}\right\}=\sum_{k=1}^{n} \int_{z_{k-1}}^{z_{k}}\left([\overline{\mathbf{Q}}]_{k}\left(\left\{\boldsymbol{\varepsilon}_{x}^{0}\right\}_{k}+z_{k}\{\mathbf{k}\}_{k}\right)\right) z d z
$$

Eq. (21) can be also written in the following form:

$$
\left\{\mathbf{M}_{x}\right\}=[\mathbf{B}]\left\{\boldsymbol{\varepsilon}_{x}^{0}\right\}+[\mathbf{D}]\left\{\mathbf{k}_{x}\right\}
$$

where:

$$
\begin{aligned}
& {[\mathbf{B}]=\frac{1}{2} \sum_{k=1}^{n}\left([\overline{\mathbf{Q}}]_{k}\left(z_{k}^{2}-z_{k-1}^{2}\right)\right)} \\
& {[\mathbf{D}]=\frac{1}{3} \sum_{k=1}^{n}\left([\overline{\mathbf{Q}}]_{k}\left(z_{k}^{3}-z_{k-1}^{3}\right)\right)}
\end{aligned}
$$

Based on Eqs. (18-23), the relationship between the mid-plane generalized forces and strain is defined as:

$$
\left\{\begin{array}{l}
\mathbf{N} \\
\mathbf{M}
\end{array}\right\}=\left[\begin{array}{ll}
\mathbf{A} & \mathbf{B} \\
\mathbf{B} & \mathbf{D}
\end{array}\right]\left\{\begin{array}{l}
\mathbf{\varepsilon}^{0} \\
\mathbf{k}
\end{array}\right\}
$$

In Eq. (24), the matrix $\mathbf{A}$ which defines the extensional behavior of the laminate, relates the mid-plane forces to the mid-plane strains. It is noted that the matrix $\mathbf{A}$ is independent of stacking sequence. The matrix $\mathbf{B}$ associates the mid-plane forces with the plate curvatures as well as mid-plane moments with mid-plane strains, and the matrix $\mathbf{B}$ is zero for symmetric 
laminates. On the other hand, the matrix $\mathbf{D}$ relates the mid-plane moments to the plate curvatures, which defines the bending behavior of the laminate. It is noted that the matrix $\mathbf{D}$ is dependent of stacking sequence, and it is most affected by the location of zero degree plies in the stacking sequence.

Eq. (24) can be expanded in the following form:

$$
\left\{\begin{array}{l}
N_{x} \\
N_{y} \\
N_{x y} \\
M_{x} \\
M_{y} \\
M_{x y}
\end{array}\right\}=\left[\begin{array}{llllll}
A_{11} & A_{12} & A_{14} & B_{11} & B_{12} & B_{14} \\
A_{12} & A_{22} & A_{24} & B_{12} & B_{22} & B_{24} \\
A_{14} & A_{24} & A_{44} & B_{14} & B_{24} & B_{44} \\
B_{11} & B_{12} & B_{14} & D_{11} & D_{12} & D_{14} \\
B_{12} & B_{22} & B_{24} & D_{12} & D_{22} & D_{24} \\
B_{14} & B_{24} & B_{44} & D_{14} & D_{24} & D_{44}
\end{array}\right]\left\{\begin{array}{c}
\varepsilon_{x}^{0} \\
\varepsilon_{y}^{0} \\
\gamma_{x y}^{0} \\
k_{x} \\
k_{y} \\
k_{x y}
\end{array}\right\}
$$

The $A_{14}$ and $A_{24}$ terms denote the extensional-shear coupling between the mid-plane forces and mid-plane shear strain, and both above relations of the matrix $\mathbf{B}$ represent the extensionalbending and shear-twist coupling. The $D_{14}$ and $D_{24}$ terms stand for the bending-twist coupling between the mid-plane moments and plate curvatures.

\section{Analysis of laminate acoustic metamaterials for elastic wave absorption}

\subsection{Dispersion analysis of laminate acoustic metamaterials with symmetric laminate}

In this section, the laminate acoustic metamaterials are proposed, which consist of two parallel orthotropic laminates and a periodic array of mass-spring-damper subsystems integrated between the two laminates, as shown in Fig. 3. In the calculation of dispersion relations, the considered structure refers to an infinite system. According to periodicity, only one unit cell (see Fig. 4) needs to be considered to investigate elastic wave absorption in laminate acoustic metamaterials regardless of boundary conditions and size effects. Its edge lengths along $x, y$ and $z$ direction are $2 a=0.3 \mathrm{~m}, 2 b=0.06 \mathrm{~m}$ and $h=0.004 \mathrm{~m}$ respectively. Two springs are set to the same 
spring constant $k$, the absorber mass is $2 m=80 \mathrm{~g}, \frac{1}{2 \pi} \sqrt{\frac{2 k}{2 m}}=400 \mathrm{~Hz}$, and both laminates move in phase and have the same magnitude of displacement. T300/5208 is selected as the material of the laminate: $\quad E_{1}=181 \mathrm{GPa}, \quad E_{2}=10.3 \mathrm{GPa}, \quad E_{3}=10.3 \mathrm{GPa}, \quad \mu_{12}=0.28, \quad \rho=1600 \mathrm{~kg} / \mathrm{m}^{3}, \quad G_{12}=7.17 \mathrm{GPa}$, $G_{13}=7.17 \mathrm{GPa}, G_{23}=3.87 \mathrm{Gpa}, X_{\mathrm{t}}=1500 \mathrm{MPa}, X_{\mathrm{c}}=1500 \mathrm{MPa}, Y_{\mathrm{t}}=40 \mathrm{MPa}, Y_{\mathrm{c}}=246 \mathrm{MPa}, S=68 \mathrm{MPa}$.

As shown in Fig. 5, the equilibrium equation of one ply can be derived as:

$$
\frac{\partial \sigma_{x}}{\partial x}+\frac{\partial \tau_{x y}}{\partial y}+\frac{\partial \tau_{x z}}{\partial z}=0
$$

In the $y$ direction

$$
\frac{\partial \tau_{y x}}{\partial x}+\frac{\partial \sigma_{y}}{\partial y}+\frac{\partial \tau_{y z}}{\partial z}=0
$$

In the $z$ direction

$$
\frac{\partial \tau_{z x}}{\partial x}+\frac{\partial \tau_{z y}}{\partial y}+\frac{\partial \sigma_{z}}{\partial z}=0
$$

Integration of Eq. (26) can be obtained with the condition of $\left.\tau_{x z}\right|_{z=\frac{h}{2}}=0$ and $\left.\tau_{x z}\right|_{z=\frac{-h}{2}}=0$ :

$$
\frac{\partial N_{x}}{\partial x}+\frac{\partial N_{x y}}{\partial y}=0
$$

Conducting the same procedure for Eq. (27), it can be expressed as:

$$
\frac{\partial N_{x y}}{\partial x}+\frac{\partial N_{y}}{\partial y}=0
$$

In the same way, the integration of Eq. (26) and Eq. (27) multiplied by $z$ give: 


$$
\begin{aligned}
& \frac{\partial M_{x}}{\partial x}+\frac{\partial M_{x y}}{\partial y}-Q_{x}=0 \\
& \frac{\partial M_{x y}}{\partial x}+\frac{\partial M_{y}}{\partial y}-Q_{y}=0
\end{aligned}
$$

where $\int_{\frac{-t}{2}}^{\frac{t}{2}} \tau_{x z} d z=Q_{x}, \int_{\frac{-t}{2}}^{\frac{t}{2}} \tau_{y z} d z=Q_{y}$.

Firstly, we can assume that $u_{x x}=\frac{\partial^{2} u}{\partial x^{2}} u_{x y}=\frac{\partial^{2} u}{\partial x \partial y}$. Based on Eq. (22) and Eqs. (31-32), we use $M_{1}, M_{2}, M_{6}, Q_{1}$ and $Q_{2}$ to redefine the moment resultants $M_{x}, M_{y}, M_{x y}, Q_{x}$ and $Q_{y}$ as:

$$
\begin{aligned}
& M_{1}=\left[B_{11} u_{x}^{0}+B_{12} v_{y}^{0}+B_{16}\left(u_{y}^{0}+v_{x}^{0}\right)\right]-\left(D_{11} w_{x x}+D_{12} w_{y y}+2 D_{16} w_{x y}\right) \\
& M_{2}=\left[B_{12} u_{x}^{0}+B_{22} v_{y}^{0}+B_{26}\left(u_{y}^{0}+v_{x}^{0}\right)\right]-\left(D_{12} w_{x x}+D_{22} w_{y y}+2 D_{26} w_{x y}\right) \\
& M_{6}=\left[B_{16} u_{x}^{0}+B_{26} v_{y}^{0}+B_{66}\left(u_{y}^{0}+v_{x}^{0}\right)\right]-\left(D_{16} w_{x x}+D_{26} w_{y y}+2 D_{66} w_{x y}\right) \\
& Q_{1}=M_{1 x}+M_{6 y} \\
& Q_{2}=M_{6 x}+M_{2 y}
\end{aligned}
$$

where $w$ is the displacement of the laminate in the $z$-axis.

In order to avoid the undesirable behaviors such as extensional-bending and shear-twist coupling in engineering, the symmetrical laminate may be the best option. As the symmetric laminate is composed of piles, both geometric (theta and thickness) and material properties are symmetrical about the middle surface of the laminate as $[0 / 45 / 90 /-45]_{s}$. Therefore, $B_{i j}=0$. Eq. (33) can be simplified as follows: 


$$
\begin{aligned}
& M_{1}=-\left(D_{11} w_{x x}+D_{12} w_{y y}+2 D_{16} w_{x y}\right) \\
& M_{2}=-\left(D_{12} w_{x x}+D_{22} w_{y y}+2 D_{26} w_{x y}\right) \\
& M_{6}=-\left(D_{16} w_{x x}+D_{26} w_{y y}+2 D_{66} w_{x y}\right) \\
& Q_{1}=M_{1 x}+M_{6 y} \\
& Q_{2}=M_{6 x}+M_{2 y}
\end{aligned}
$$

Then the kinetic energy $\delta T$, elastic strain energy $\delta U$ and non-conservative work $\delta W_{n c}$ of the laminate can be represented as:

$$
\delta T=\int_{-a}^{a} \int_{-b}^{b}(\rho t \ddot{w} \delta w) d x d y
$$

$$
\begin{aligned}
& \delta U=\int_{-a}^{a} \int_{-b}^{b} \int_{-t / 2}^{t / 2}\left(\sigma_{x} \delta \varepsilon_{x}+\sigma_{y} \delta \varepsilon_{y}+\sigma_{x y} \delta \varepsilon_{x y}\right) d z d x d y \\
& =\int_{-a}^{a} \int_{-b}^{b} \int_{-t / 2}^{t / 2}\left(-M_{1 x x} \delta w-M_{2 y y} \delta w-M_{6 x y} \delta w\right) d x d y
\end{aligned}
$$

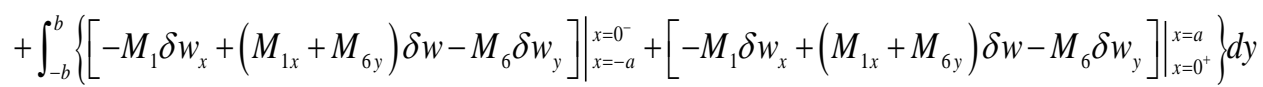

$$
\begin{aligned}
& +\int_{-a}^{a}\left\{\left.\left[-M_{2} \delta w_{y}+\left(M_{2 y}+M_{6 x}\right) \delta w-M_{6} \delta w_{x}\right]\right|_{y=-b} ^{y=0^{-}}+\left.\left[-M_{2} \delta w_{y}+\left(M_{2 y}+M_{6 x}\right) \delta w-M_{6} \delta w_{x}\right]\right|_{x=0^{+}} ^{x=b}\right\} d x \\
& \delta W_{n c}=\left.\int_{-b}^{b}\left[-M_{1} \delta w_{x}+Q_{1} \delta w-M_{6} \delta w_{y}\right]\right|_{x=-a} ^{x=a} d y \\
& +\left.\int_{-a}^{a}\left[-M_{2} \delta w_{y}+Q_{2} \delta w-M_{6} \delta w_{x}\right]\right|_{y=-b} ^{y=b} d x+k\left(u^{\prime}-w_{0}\right) \delta w_{0}
\end{aligned}
$$

According to Hamilton principle, we can obtain the following formula:

$$
\int_{0}^{t}\left(\delta T-\delta U+\delta W_{n c}\right) d t=0
$$

Substituting Eqs. (35-37) into Eq. (38)

$$
\begin{aligned}
& \int_{0}^{t}\left\{\int_{-a}^{a} \int_{-b}^{b}\left(-\rho h \ddot{w}+M_{1 x x}+M_{2 y y}+2 M_{6 x y}+\left[\bar{Q}+k\left(u^{\prime}-w_{0}\right)\right] \delta(x, y)\right) \delta w d x d y\right\} d t=0 \\
& \bar{Q}=\left(Q_{1}^{x=\varepsilon_{x} / 2}-Q_{1}^{x=-\varepsilon_{x} / 2}\right) \varepsilon_{y}+\left(Q_{2}^{y=\varepsilon_{y} / 2}-Q_{1}^{y=-\varepsilon_{y} / 2}\right) \varepsilon_{x}, \varepsilon_{x}, \varepsilon_{y} \approx 0
\end{aligned}
$$


where $\delta(x, y)$ is a 2D Dirac delta function, and $\bar{Q}$ represents the discontinuity of the internal transverse shear force at the absorber location.

We can obtain the governing equation of the laminate by setting the coefficient of $\delta w$ to zero

$$
-\rho h \ddot{w}+M_{1 x x}+M_{2 y y}+2 M_{6 x y}+\left[\bar{Q}+k\left(u^{\prime}-w_{0}\right)\right] \delta(x, y)=0
$$

We assume the upper laminate as a rigid body which moves with the average acceleration on the area $2 a \times 2 b$. In addition, the upper laminate is subjected to lateral shear force on the four edges and the concentrated force from the absorber. Thus, the $\bar{Q}$ can be ignored. Then integrate Eq. (39) on the upper laminate:

$$
\begin{aligned}
& \int_{-a}^{a} \int_{-b}^{b}\left\{-\rho h \ddot{w}+M_{1 x x}+M_{2 y y}+2 M_{6 x y}+\left[\bar{Q}+k\left(u^{\prime}-w_{0}\right)\right] \delta(x, y)\right\} d x d y \\
& =\int_{-a}^{a} \int_{-b}^{b}(-\rho h \ddot{w}) d y d x+\left.\int_{-a}^{a}\left(M_{2 y}+M_{6 x}\right)\right|_{y=-b} ^{y=b} d x \\
& \quad+\left.\int_{-b}^{b}\left(M_{1 x}+M_{6 y}\right)\right|_{x=-a} ^{x=a} d y+k\left(u^{\prime}-w_{0}\right)
\end{aligned}
$$

The governing equation of the vibration absorber can be easily obtained from Newton's Second Law as:

$$
m \ddot{u}^{\prime}=k\left(w_{0}-u^{\prime}\right)
$$

If a $2 D$ elastic wave at specific frequency propagates within infinite laminate acoustic metamaterials made of a periodic unit cell (see Fig. 4(b)), the laminate's displacement $w$ and the absorber's displacement $u^{\prime}$ can be assumed to have the following forms:

$$
w=p e^{j(\alpha x+\beta y-\omega t)}, u^{\prime}=q e^{-j \omega t}
$$


where $\alpha\left(\equiv 2 \pi / \lambda_{1}\right)$ and $\beta\left(\equiv 2 \pi / \lambda_{2}\right)$ are the wave numbers along the $x$ and $y$ directions with $\lambda_{1}$ and $\lambda_{2}$ being the corresponding wave lengths, $\omega$ is the wave frequency, and $p$ and $q$ are the displacement amplitudes.

Substitute Eq. (43) into Eqs. (41) and (42), and rewrite the results in a matrix form:

$$
\left[\begin{array}{cc}
\frac{4 \sin (a \alpha) \sin (b \beta)}{\alpha \beta}\left\{\rho h w^{2}-\left[D_{11} \alpha^{4}+2\left(D_{12}+2 D_{66}\right) \alpha^{2} \beta^{2}+D_{22} \beta^{4}+4 D_{26} \alpha \beta^{3}+4 D_{16} \alpha^{3} \beta\right]\right\}-k & k \\
k & m \omega^{2}-k
\end{array}\right]\left[\begin{array}{l}
p \\
q
\end{array}\right]=0
$$

To have non-zero solutions in the eigenvalue problem shown in Eq. (44), the determinant of the matrix needs to be zero and the dispersion equation that relates $\omega$ to $\alpha$ and $\beta$ is obtained as:

$$
\left(m \omega^{2}-k\right)\left[\frac{4 \sin (a \alpha) \sin (b \beta)}{\alpha \beta}\left\{\rho h w^{2}-\left[D_{11} \alpha^{4}+2\left(D_{12}+2 D_{66}\right) \alpha^{2} \beta^{2}+D_{22} \beta^{4}+4 D_{26} \alpha \beta^{3}+4 D_{16} \alpha^{3} \beta\right]\right\}-k\right]-k^{2}=0
$$

The upper bound of the stopband is obtained from the upper dispersion surface with $\alpha$ and $\beta \rightarrow 0$, and the lower bound can be determined from the lower dispersion surface with $\alpha$ and $\beta \rightarrow \infty$, as shown in Fig. 6(a).

$$
\text { Stopband }=\left(\frac{1}{2 \pi} \sqrt{k / m}, \frac{1}{2 \pi} \sqrt{k / m+k /(4 a b t \rho)}\right)
$$

where $4 a b t \rho$ is the mass of the laminate's unit cell.

Fig. 6(b) shows a perspective view of the dispersion surface. There is a stopband between $400 \mathrm{~Hz}$ and $464.5 \mathrm{~Hz}$. Eq. (45) shows that the width of stopband can be increased by reducing the ratio $4 a b t \rho / m$. Although the stopband derivation results of symmetric laminate acoustic metamaterials are the same as the metamaterial plates in Ref ${ }^{[21]}$, the laminate acoustic metamaterials are able to have much wider stopband than conventional metamaterial plates on account of the high strength to weight ratio of CFRP. The stopband derived above is based on infinite laminate acoustic metamaterials. Thus, finite element modeling as well as frequency 
response analysis is needed to validate the performance of finite laminate acoustic metamaterials, which is studied in the next section.

\subsection{Frequency response analysis of laminate acoustic metamaterials}

In this section, numerical examples of the laminate acoustic metamaterials are studied, and the material of the laminates is T300/520: $E_{1}=181 \mathrm{GPa}, E_{2}=10.3 \mathrm{GPa}, E_{3}=10.3 \mathrm{GPa}, \mu_{12}=0.28$, $\rho=1600 \mathrm{~kg} / \mathrm{m}^{3}, \quad G_{12}=7.17 \mathrm{GPa}, \quad G_{13}=7.17 \mathrm{GPa}, \quad G_{23}=3.87 \mathrm{Gpa}, \quad X_{\mathrm{t}}=1500 \mathrm{MPa}, \quad X_{\mathrm{c}}=1500 \mathrm{MPa}$, $Y_{\mathrm{t}}=40 \mathrm{MPa}, Y_{\mathrm{c}}=246 \mathrm{MPa}, S=68 \mathrm{MPa}$. In order to consider the effects of boundary conditions and damping ratios of the vibration absorbers on laminates, the laminate acoustic metamaterials shown in Fig. 7(a) is considered, which has vertical length $L_{a}=6 \mathrm{~m}$ and horizontal length $L_{b}=3.6 \mathrm{~m}$. For the laminate, we choose the symmetric laminate which is composed of piles such that both geometric (theta and thickness) and material properties are symmetrical about the middle surface of the laminate as $[0 / 45 / 90 /-45]_{s}$. The thickness of each ply is set to be $h=0.5 \mathrm{~mm}$. The resonant frequency of the absorber is set to be $f=400 \mathrm{~Hz}$. The mass of the absorber is set to be $2 m=80 \mathrm{~g}$. The total mass of the subsystems is $24 \%$ of the total mass of the laminate acoustic metamaterials. The distance between the top and bottom laminates is $200 \mathrm{~mm}$.

The two vertical edges at $x=0$ and $6 \mathrm{~m}$ are hinged, and a white noise excitation with unit load in the $z$ direction is applied at the nodes with the coordinate of $(60,1800,0)$ and $(60,1800,200)$ (i.e., the green dot in Fig. 7(b)). Each laminate is modeled by $100 \times 12$ four-node rectangular plate elements. The blue dots at the element nodes represent the single-frequency absorbers. There are no absorbers on the hinged edges at $x=0$ and $6 \mathrm{~m}$ and the free edges at $y=-1.8$ and $1.8 \mathrm{~m}$. The unit cell here has the same parameters as those used in the dispersion analysis presented in Fig. 4. 
We assume that the same external excitation is loaded on the same position of the two laminates. Hence, both laminates move in phase and have the same magnitude of displacement. The frequency response analysis (FRA) based on modal superposition method is conducted through commercial software Hyperworks, and two representative frequency response functions (FRFs) under different damping ratio of the laminate acoustic metamaterials are studied and plotted in Fig. 8(a) and Fig. 8(b). The black lines represent the laminate acoustic metamaterials without vibration absorbers for reference. The blue lines show FRFs of laminate acoustic laminates with the damping ratio increasing from 0.001 to 0.1 . The red lines show a stopband to the right side of $400 \mathrm{~Hz}$ with a low damping ratio $\xi=0.001$ for each vibration absorber, which agrees well with the results of dispersion analysis. The stopband between $400 \mathrm{~Hz}$ and $464.5 \mathrm{~Hz}$ exists, which is nearly twice as wide as the stopband in the conventional metamaterials plate. The ability to obtain such a wide band gap is due to the fact that, the laminate acoustic metamaterial with superior strength to weight ratio can achieve the high ratio of vibrationabsorber-mass/unit-laminate-cell-mass without increasing the overall mass of the structure significantly.

The key working mechanism of the bandgaps is that when the excitation frequency is close to the frequency range of stopband, the vibration absorbers approach the resonance at the same time, and there are many frequency response peaks around the stopband as shown in Fig. 8 . The incoming elastic wave is affected strongly by resonance scattering of the vibration absorbers. Therefore, the energy of incoming elastic wave is attenuated efficiently along the direction of propagation. Furthermore, with an appropriate damping ratio of the vibration absorbers, the vibration of laminate acoustic metamaterials can be suppressed effectively within the stopband. 
Moreover, the stopband can be widened through increasing the damping ratio of vibration absorbers, as shown in Fig.8. (the blue lines)

\section{Design for the vehicle door composed of laminate acoustic metamaterials}

In this section, we design the vehicle door composed of low-frequency laminate acoustic metamaterials with a wide stopband. The vibration of the structure can be suppressed by selecting a suitable mass-spring-damper system and then arranging them on laminate acoustic metamaterials properly. We can change the natural frequency of vibration absorber by adjusting the spring constant while maintaining the mass of vibration absorber. There are many factors that affect the wave propagation in laminate acoustic metamaterials, so we should take the laminate's boundary conditions, low-order natural frequencies, vibration modes of the structures, the absorber's resonant frequencies and locations into consideration.

A simple model of the vehicle door composed of laminate acoustic metamaterials and the mesh are shown in Figs. 9(a) and 9(b), respectively. The laminate acoustic metamaterials consist of two parallel orthotropic laminates and a periodic array of mass-spring vibration absorbers integrated between the two laminates (see Fig. 4). The dimensions of each laminate are $0.95 \mathrm{~m}$ in the $x$-direction, $11.5 \mathrm{~m}$ in the $y$-direction, $8 \mathrm{~mm}$ in the $z$-direction respectively. The material of the laminates $\mathrm{T} 300 / 5208$ is selected: $E_{1}=181 \mathrm{GPa}, \quad E_{2}=10.3 \mathrm{GPa}, \quad \mu_{12}=0.33, \quad \rho=1600 \mathrm{~kg} / \mathrm{m}^{3}$, $G_{12}=7.17 \mathrm{GPa}, \quad G_{13}=7.17 \mathrm{GPa}, \quad G_{23}=3.87 \mathrm{Gpa}, \quad X_{\mathrm{t}}=1500 \mathrm{MPa}, \quad X_{\mathrm{c}}=1500 \mathrm{MPa}, \quad Y_{\mathrm{t}}=40 \mathrm{MPa}$, $Y_{\mathrm{c}}=246 \mathrm{MPa}, S=68 \mathrm{MPa}$. The laminates are symmetric as $[0 / 45 / 90 /-45]_{s}$ and the mass of each vibration absorber is designed to be $2 \mathrm{~g}$. The distance between the top and bottom laminates is $12 \mathrm{~mm}$.

The two hinges and bolt of the door are hinged here (i.e., red dots in Fig. 9(b)), and a white noise excitation with amplitude of $1 \mathrm{kN}$ is applied in the area of the door's hinge (i.e., green dots 
in Fig. 9(b)) on both laminates. As shown in Fig. 9(b), each laminate is modeled by four-node rectangular plate elements with the mesh size of $10 \times 10$. As is known, the high frequency noise in the vehicle can be reduced effectively by the sound-absorbing material, but the low frequency noise is difficult to control due to the longer wavelength. On the other hand, the frequency range of the low frequency noise is mainly controlled by the car engine. In general, the main excitation frequency of the car engine is under $200 \mathrm{~Hz}$. Thus, FRA is conducted first to find the peak response of laminate acoustic metamaterials without vibration absorbers. The black lines in Fig. 10 represent the FRFs of the center and corner nodes of the laminate acoustic metamaterials without vibration absorbers. The first natural frequency is $27 \mathrm{~Hz}$ and second natural frequency is around $60 \mathrm{~Hz}$, the third natural frequency is around 144Hz. Figs. 11(a)-(f) show the FRA of the low-frequency laminate acoustic metamaterials without vibration absorbers. The peak response of low-frequency bands can be lowered by locating vibration absorbers with specific frequency appropriately on the basis of natural frequencies and mode shapes of the laminate acoustic metamaterials.

The FRA at $25 \mathrm{~Hz}$ and $30 \mathrm{~Hz}$ in Figs. 11 (a) and 11 (b) indicates that the large vibration amplitudes appear around the bottom right corner of the vehicle door. Hence the first group of vibration absorbers with a resonant frequency of $28 \mathrm{~Hz}$ (i.e., green dots in Fig. 12) is placed around the right corner. The FRA at $55 \mathrm{~Hz}$ and $60 \mathrm{~Hz}$ in Figs. 11(c) and 11(d) shows that the large vibration amplitudes appear around the window of the vehicle door and the second group of vibration absorbers with a resonant frequency of $58 \mathrm{~Hz}$ (i.e., blue dots in Fig. 12) is placed around the window. The FRA at $145 \mathrm{~Hz}$ and $150 \mathrm{~Hz}$ in Figs. 11(e) and 11(f) illustrates that the large vibration amplitudes appear around the door frames and the bottom left corner of the vehicle door, so the third group of vibration absorbers with a resonant frequency of $148 \mathrm{~Hz}$ (i.e., purple 
dots in Fig. 12) is placed around the door frames and left corner. After adding the three groups of absorbers, the total mass of the vibration absorbers is $33 \%$ of the laminate acoustic metamaterials.

FRA is conducted again in the vehicle door after the proper design of laminate acoustic metamaterials. Figs. 13(a)-(d) indicate the FRA of the vehicle door at $25 \mathrm{~Hz}, 55 \mathrm{~Hz}$ and $145 \mathrm{~Hz}$, $150 \mathrm{~Hz}$ with vibration absorbers. In contrast to the FRA without absorber as shown in Figs. 11(a), 11(c) and 11(e)-(f), the vibration amplitude contours have clearly illustrated that the big vibration around the vehicle door has been suppressed effectively, which has strongly demonstrated the excellent performance of laminate acoustic metamaterials. Although the above results from FRA are obtained in the main engine operating frequency range, it is straightforward to design laminate acoustic metamaterials to suppress the high frequency vibration based on the same principle.

\section{Conclusions}

This paper presents a new laminate acoustic metamaterial designed by integrating two parallel orthotropic laminates, which is composed of carbon-fiber-reinforced polymer (CFRP), with a periodic array of mass-spring-damper subsystems. Based on the concept of conventional vibration, the band gap of laminate acoustic metamaterials has been derived by dispersion analysis in this work. For the first time, it is found that the laminate acoustic metamaterials are able to have wider stopband than conventional metamaterial plates. Numerical results reveal that the stopband's location depends on the local resonant frequency of vibration absorbers, and the stopband's width is determined by the vibration-absorber-mass/unit-laminate-cell-mass ratio. On the other hand, finite-element modeling and frequency response analysis are performed to validate the theoretical analysis. In the design of the vehicle door composed of low-frequency laminate acoustic metamaterials for vibration suppression, the resonant frequencies, the 
distributions and locations of vibration absorbers are determined based on low-order natural frequencies and mode shapes of the laminate acoustic metamaterials. The simulation has verified that the laminate acoustic metamaterials with the superior strength to weight ratio of CFRP are very effective to suppress the vibration of vehicle door.

\section{Acknowledgement}

The project is supported by the Project funded by China Postdoctoral Science Foundation.

The authors also wish to thank Research Project of the Science Fund of State Key Laboratory of Advanced Design and Manufacturing for Vehicle Body (Grant No. 51375001 and 31615002), Research Project of State Key Laboratory of Mechanical Systems and Vibration (MSV 201613 and MSV201711), and Open Foundation of the State Key Laboratory of Fluid Power and Mechatronic Systems GZK F-201601.

\section{References}

[1] Veselago VG. Electrodynamics of Media with Simultaneously Negative Electric Permittivity and Magnetic Permeability2002.

[2] Walser ARM. Electromagnetic metamaterials. Proceedings of SPIE - The International Society for Optical Engineering. 2001;33(10):931-4.

[3] Nefedov IS, Tretyakov SA. Photonic band gap structure containing metamaterial with negative permittivity and permeability. Physical Review E Statistical Nonlinear \& Soft Matter Physics. 2002;66(3 Pt 2B):036611.

[4] Haberman MR, Guild MD. Acoustic metamaterials. MRS Bulletin. 2008;69(10 (Negative-Index Materials)):931-4.

[5] Pai PF. Metamaterial-based Broadband Elastic Wave Absorber. Journal of Intelligent Material Systems \& Structures. 2010;21(5):517-28.

[6] Pai PF, Peng H, Jiang S. Acoustic metamaterial beams based on multi-frequency vibration absorbers. International Journal of Mechanical Sciences. 2014;79(1):195-205. 
[7] Sun H, Du X, Pai PF. Theory of Metamaterial Beams for Broadband Vibration Absorption. Journal of Intelligent Material Systems \& Structures. 2010;21(11):1085-101.

[8] Lee SH, Park CM, Seo YM, Wang ZG, Kim CK. Composite acoustic medium with simultaneously negative density and modulus. Physical Review Letters. 2010;104(5):054301.

[9] Zhang S, Park YS, Li J, Lu X, Zhang W, Zhang X. Negative refractive index in chiral metamaterials. Physical Review Letters. 2009;102(2):023901.

[10] Zhang S. Acoustic metamaterial design and applications. Dissertations \& Theses Gradworks. 2010.

[11] Zhu R, Huang GL, Huang HH, Sun CT. Experimental and numerical study of guided wave propagation in a thin metamaterial plate. Physics Letters A. 2011;375(30-31):2863-7.

[12] Zhu R, Liu XN, Hu GK, Sun CT, Huang GL. A chiral elastic metamaterial beam for broadband vibration suppression. Journal of Sound \& Vibration. 2013;333(10):2759-73.

[13] Liu Z, Zhang X, Mao Y, Zhu YY, Yang Z, Chan CT, et al. Locally resonant sonic materials. Science. 2003;338(1-4):201-5.

[14] Wang G, Yu D, Wen J, Liu Y, Wen X. One-dimensional phononic crystals with locally resonant structures. Physics Letters A. 2004;327(5):512-21.

[15] Yu D, Liu Y, Wang G, Zhao H, Qiu J. Flexural vibration band gaps in Timoshenko beams with locally resonant structures. Journal of Applied Physics. 2006;100(12):124901--5.

[16] Wen J, Yu D, Gang W, Zhao H, Liu Y, Wen X. The directional propagation characteristics of elastic wave in two-dimensional thin plate phononic crystals. Journal of Physics D Applied Physics. 2007;24(41):1305-8.

[17] Ding Y, Liu Z, Qiu C, Shi J. Metamaterial with simultaneously negative bulk modulus and mass density. Physical Review Letters. 2007;99(9):093904.

[18] Phani AS, Woodhouse J, Fleck NA. Wave propagation in two-dimensional periodic lattices. Journal of the Acoustical Society of America. 2006;119(4):1995-2005.

[19] Liu XN, Hu GK, Huang GL, Sun CT. An elastic metamaterial with simultaneously negative mass density and bulk modulus. Applied Physics Letters. 2011;98(25):251907--3.

[20] Peng H, Pai PF, Deng H. Acoustic Multi-stopband Metamaterial Plates Design for Broadband Elastic Wave Absorption and Vibration Suppression. International Journal of Mechanical Sciences. 2015;103:104-14. 
[21] Peng H, Pai PF. Acoustic metamaterial plates for elastic wave absorption and structural vibration suppression. International Journal of Mechanical Sciences. 2014;89:350-61.

[22] Kushwaha MS, Halevi P, Dobrzynski L, Djafarirouhani B. Acoustic band structure of periodic elastic composites. Physical Review Letters. 1993;10(9):977-1094.

[23] Vasseur JO, Deymier PA, Frantziskonis G, Hong G, Djafarirouhani B, Dobrzynski L. Experimental evidence for the existence of absolute acoustic band gaps in two-dimensional periodic composite media. Journal of Physics Condensed Matter. 1999;10(27):6051.

[24] Sigalas M, Economou EN. Band structure of elastic waves in two dimensional systems. Solid State Communications. 1993;86(3):141-3.

[25] Zhu R, Huang GL, Hu GK. Effective Dynamic Properties and Multi-Resonant Design of Acoustic Metamaterials. Journal of Vibration \& Acoustics. 2012;134(3):031006.

[26] Koricho EG, Belingardi G. An experimental and finite element study of the transverse bending behaviour of CFRP composite T-joints in vehicle structures. Composites Part B Engineering. 2015;79(1):430-43.

[27] Yazdani N, Goucher E. Increasing durability of lightweight concrete through FRP wrap. Composites Part B Engineering. 2015;82:166-72.

[28] Subhani M, Al-Ameri R. Strength reduction in square columns confined with CFRP under marine environment. Composites Part B Engineering. 2016;97:183-92.

[29] Cheng J, Qiu J, Ji H, Wang E, Takagi T, Uchimoto T. Application of low frequency ECT method in noncontact detection and visualization of CFRP material. Composites Part B Engineering. 2017;110:141-52.

[30] Ghoshal A, Ayers J, Gurvich M, Urban M, Bordick N. Experimental Investigations in Embedded Sensing for Structural Health Monitoring of Composite Components in Aerospace Vehicles. ASME 2012 Conference on Smart Materials, Adaptive Structures and Intelligent Systems2014. p. 845-54.

[31] Galuppi L, Royer-Carfagni G. Shear coupling effects of the core in curved sandwich beams. Composites Part B Engineering. 2015;76:320-31.

[32] Wang G, Wen X, Wen J, Shao L, Liu Y. Two-dimensional locally resonant phononic crystals with binary structures. Physical Review Letters. 2004;93(15):154302. 
[33] Song Y, Wen J, Yu D, Liu Y, Wen X. Reduction of vibration and noise radiation of an underwater vehicle due to propeller forces using periodically layered isolators. Journal of Sound \& Vibration. 2014;333(14):3031-43.

[34] Shen H, Païdoussis MP, Wen J, Yu D, Cai L, Wen X. Acoustic cloak/anti-cloak device with realizable passive/active metamaterials. Journal of Physics D Applied Physics. 2012;45(28):1-7.

[35] Hui D, Dutta PK. A new concept of shock mitigation by impedance-graded materials. Composites Part B Engineering. 2011;42(8):2181-4.

[36] Jia Z, Ma HL, Cheng LK, Lau KT, Hui D, Yuan G. Stress transfer properties of carbon nanotube reinforced polymer composites at low temperature environments. Composites Part B Engineering. 2016;106:356-65.

[37] $\mathrm{Xu} \mathrm{X}, \mathrm{Xu} \mathrm{B}$, Hong C, Hui D. Effect of pyrolytic carbon interface thickness on microstructure and mechanical properties of lightweight zirconium boride modified carbonbonded carbon fiber composites. Composites Part B Engineering. 2016;96:305-11.

[38] Fang H, Xu X, Liu W, Qi Y, Bai Y, Zhang B, et al. Flexural behavior of composite concrete slabs reinforced by FRP grid facesheets. Composites Part B Engineering. 2016;92:4662.

[39] Changlei X, Jason Y, Sheldon QS, Ying Q, Liping C, Wu HF, et al. Natural fiber and aluminum sheet hybrid composites for high electromagnetic interference shielding performance. Composites Part B: Engineering. 2017:121-7.

[40] Kireitseu M, Hui D, Tomlinson G. Advanced shock-resistant and vibration damping of nanoparticle-reinforced composite material. Composites Part B Engineering. 2008;39(1):128-38. 


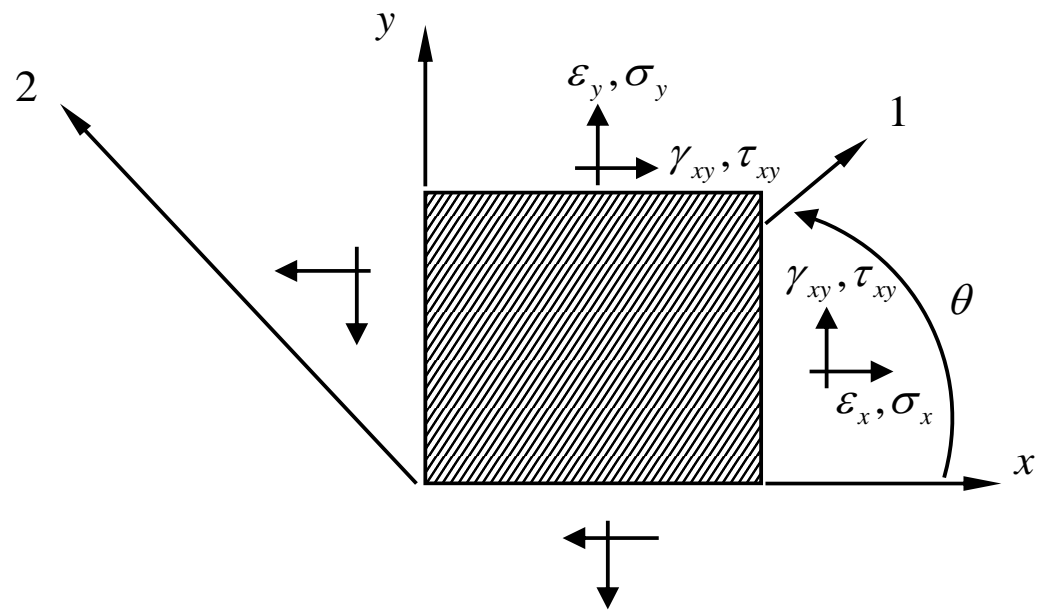

Fig. 1. Illustration of strain of a ply in the principal material 1-, 2- coordinate system and global x-, ycoordinate system 


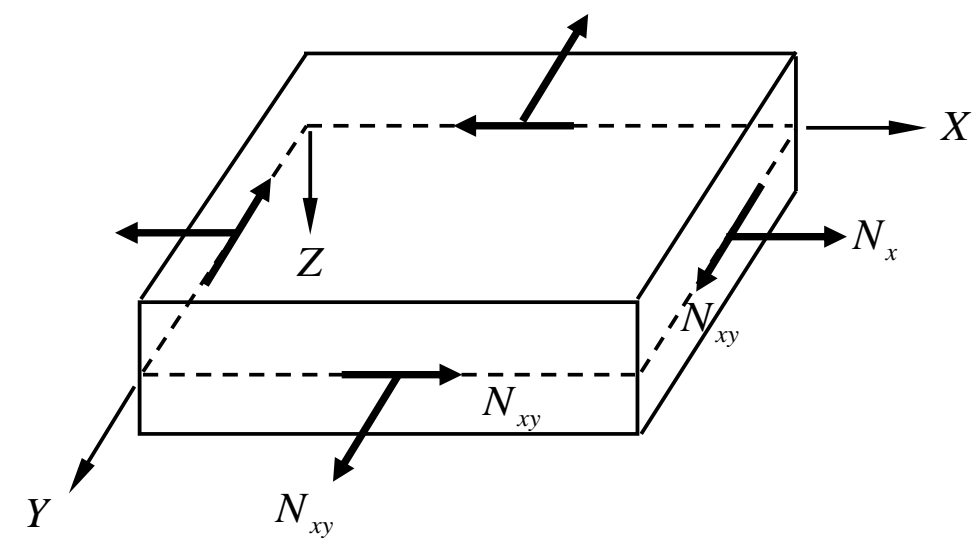

(a)

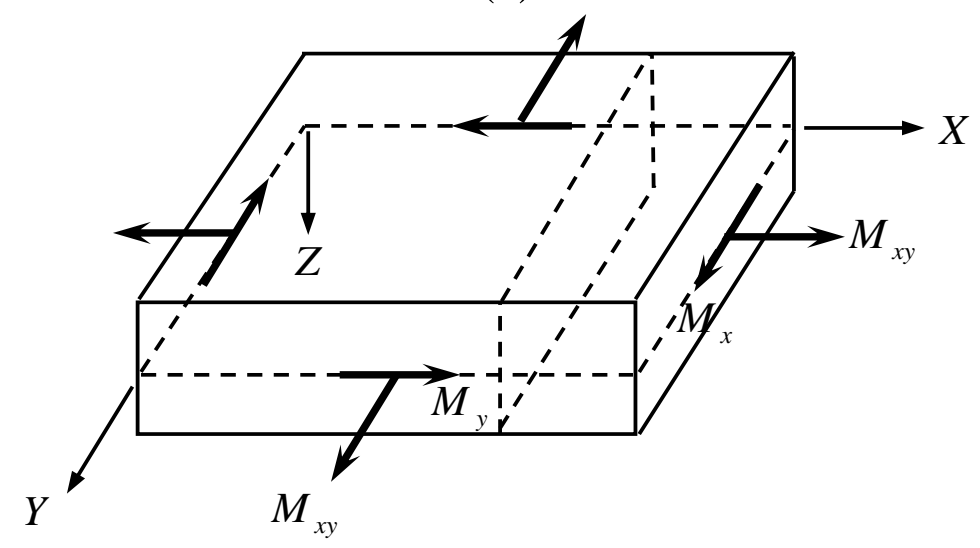

(b)

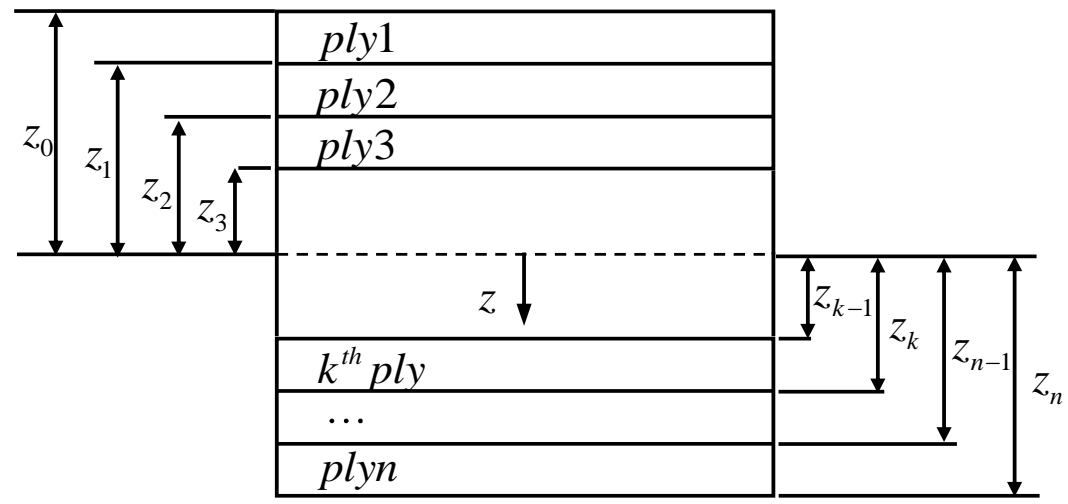

(c)

Fig. 2. The positive sign convention for laminate plate: (a) Illustration of positive Mid-Plane Forces and $(b)$ Illustration of positive Mid-Plane Moments, $(c)$ Coordinate locations of piles in a laminate 


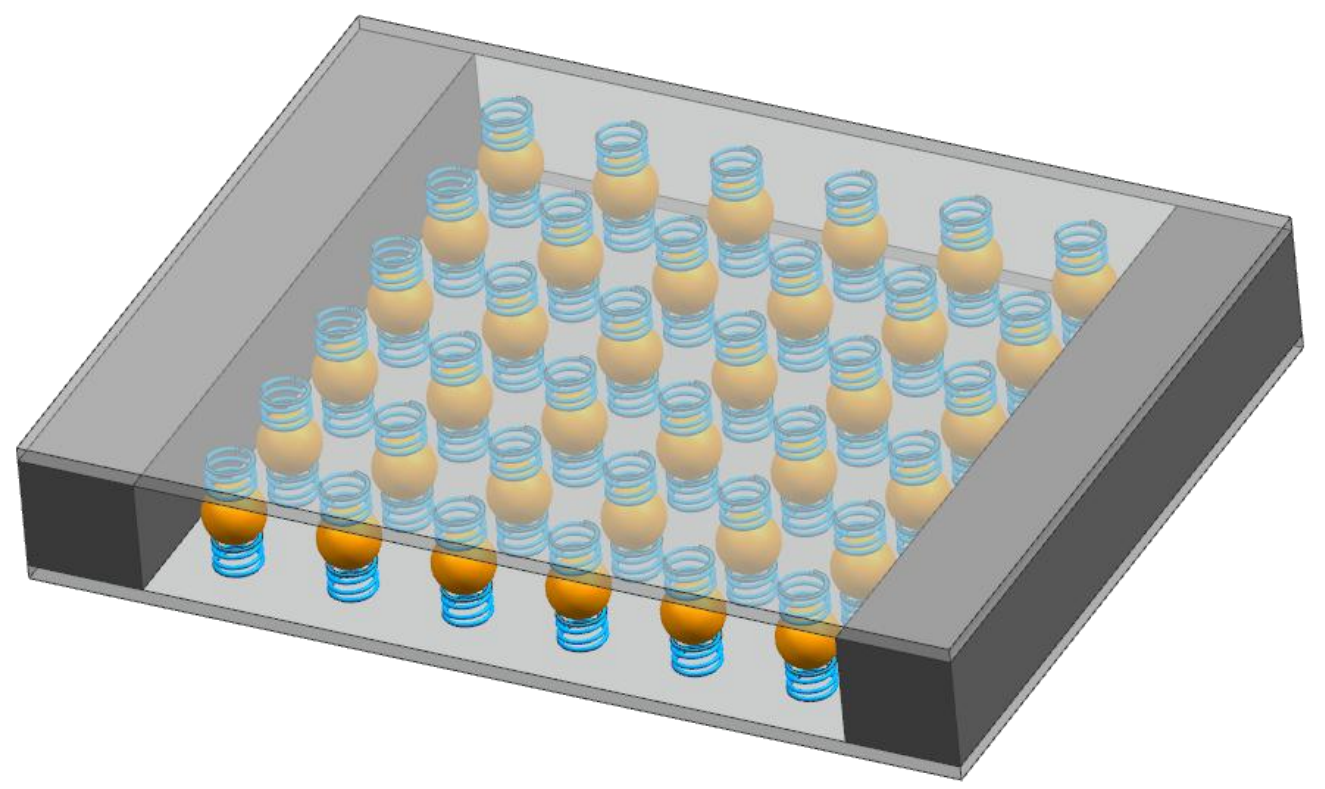

Fig. 3. A laminate metamaterial with spring-mass subsystems 


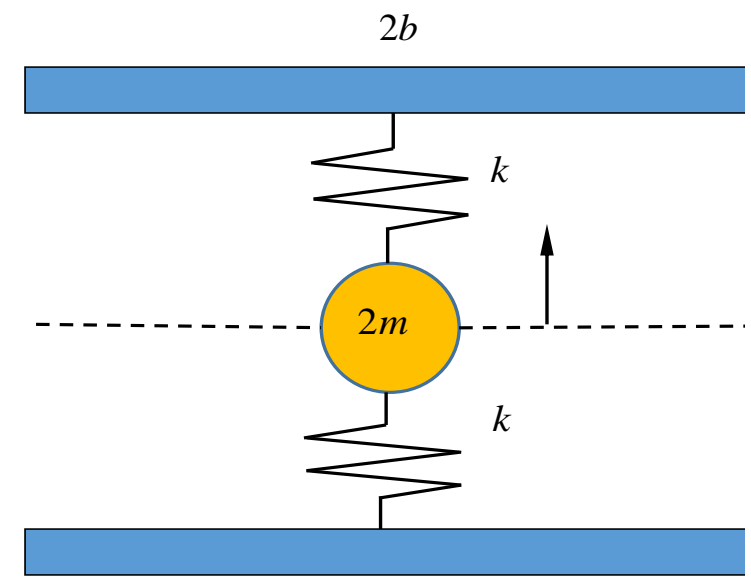

$2 b$

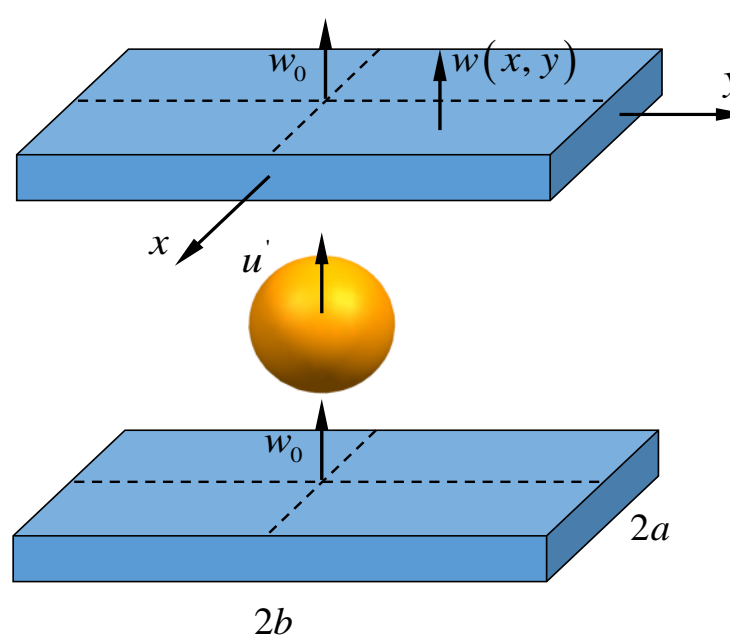

(b)

Fig. 4. A unit cell of the laminate metamaterial: (a) front view, and $(b)$ perspective view 


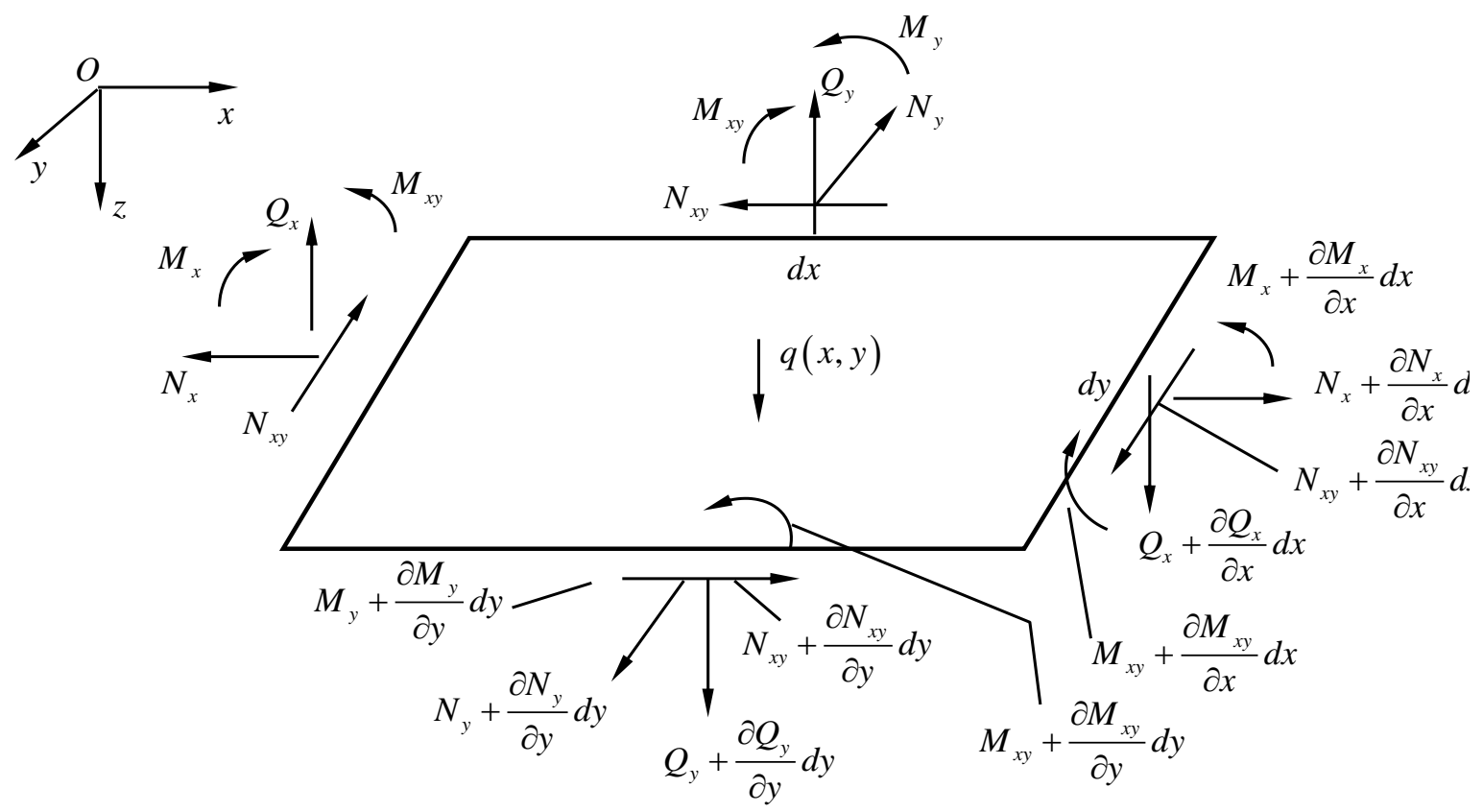

Fig. 5. Illustration of positive Mid-Plane forces and Moments of the laminate element. 


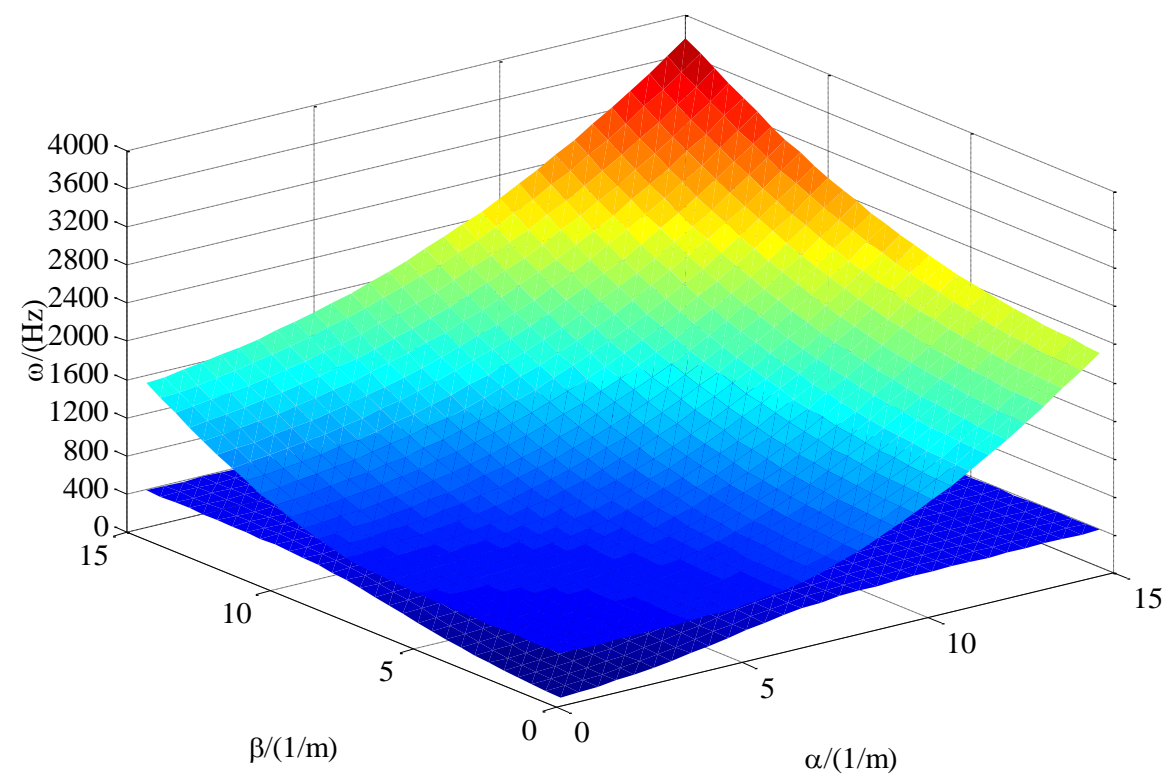

(a)

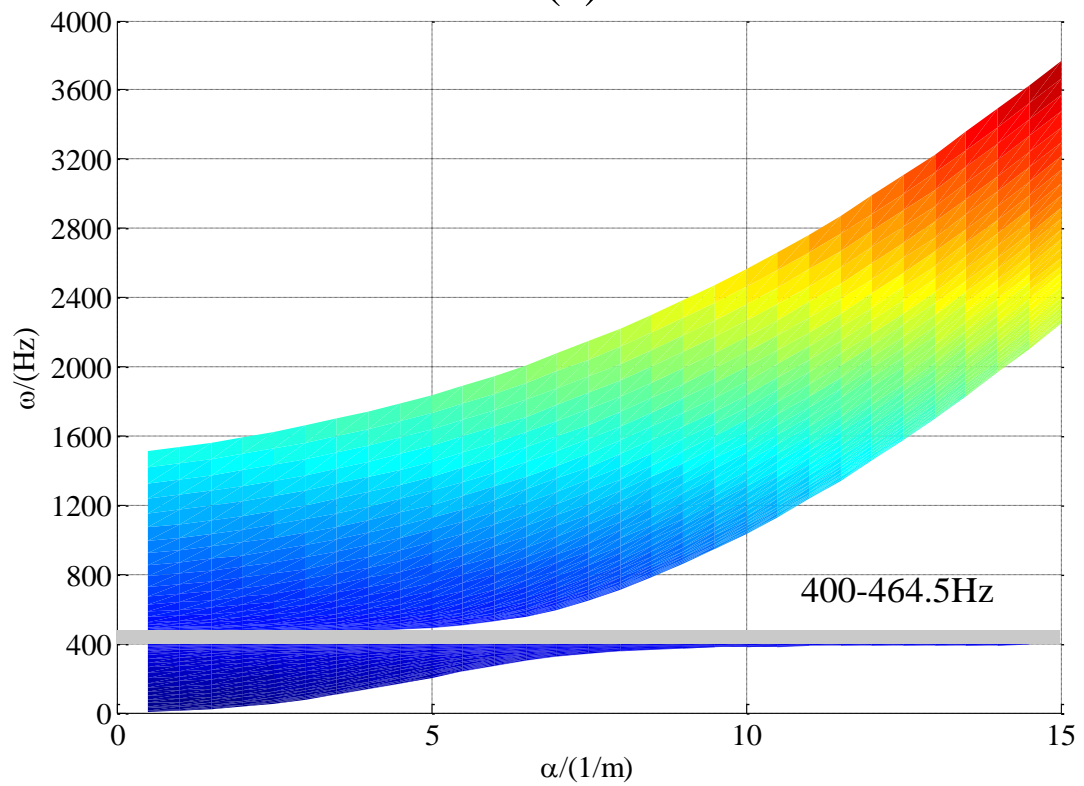

(b) 
Fig. 6. Dispersion surfaces and stopband: (a) dispersion surfaces, and (b) stopband (gray rectangle).

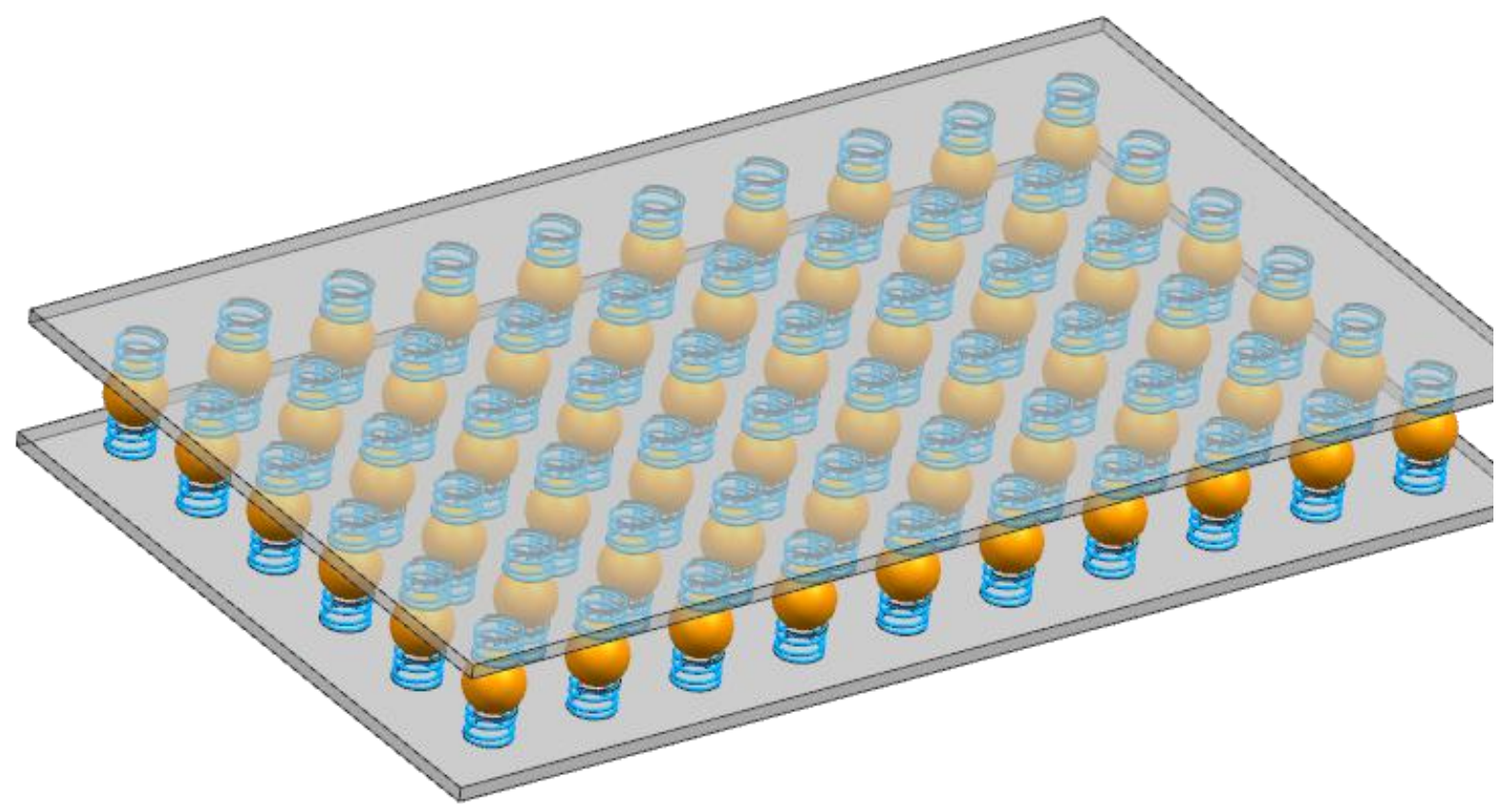

(a) 


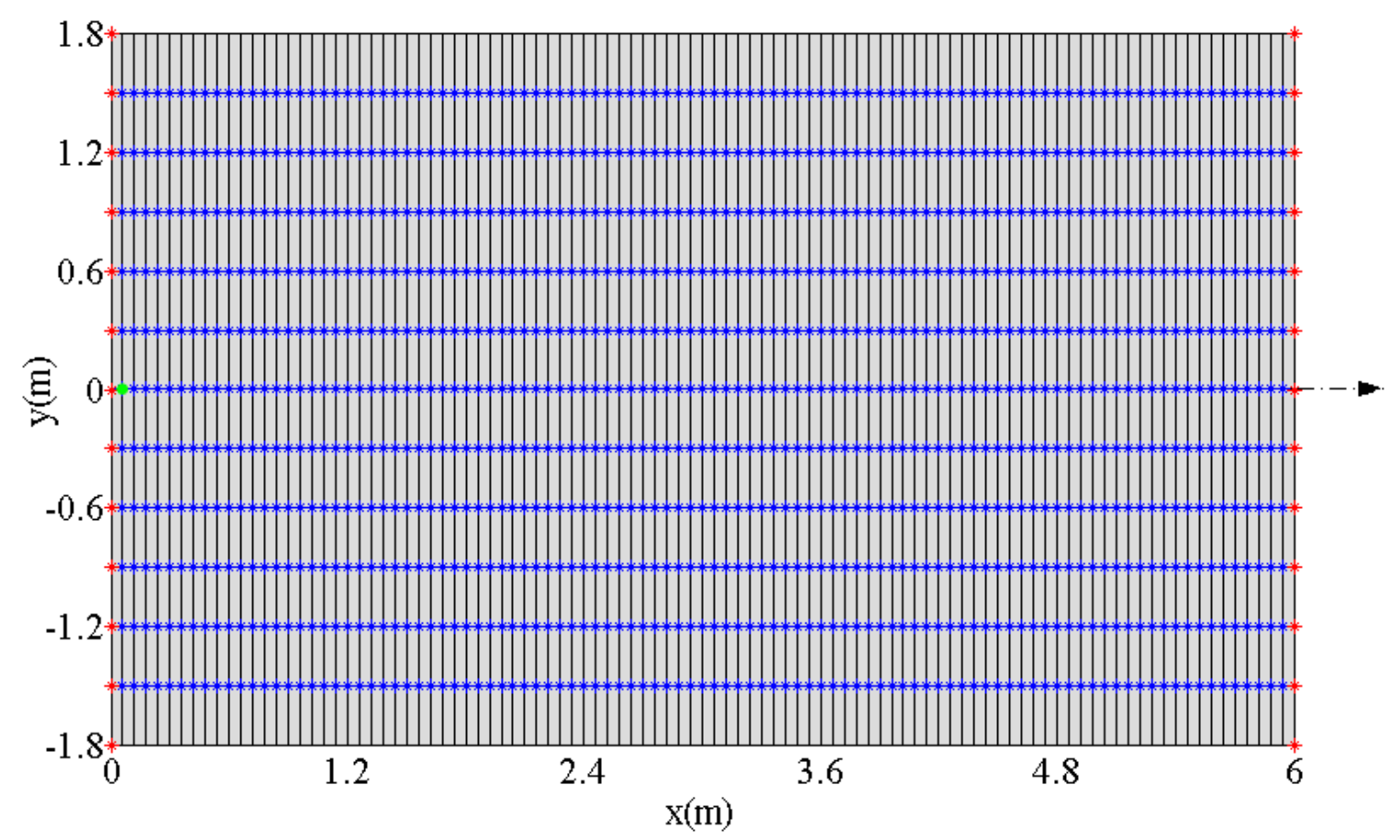

(b)

Fig. 7. A metamaterial laminate with two edge hinged: $(a)$ a 3D model, and $(b)$ a finite-element model.

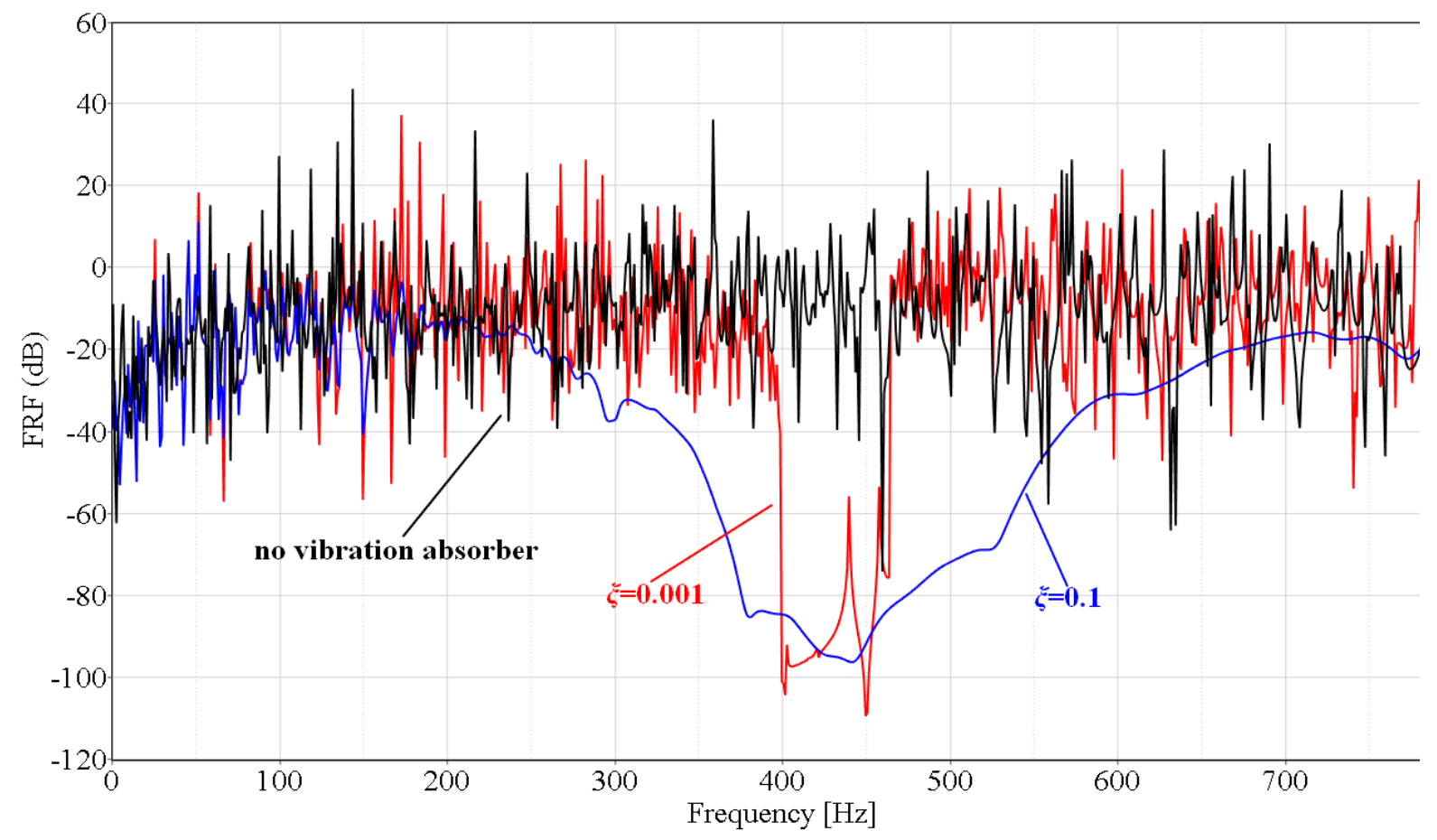

(a) 


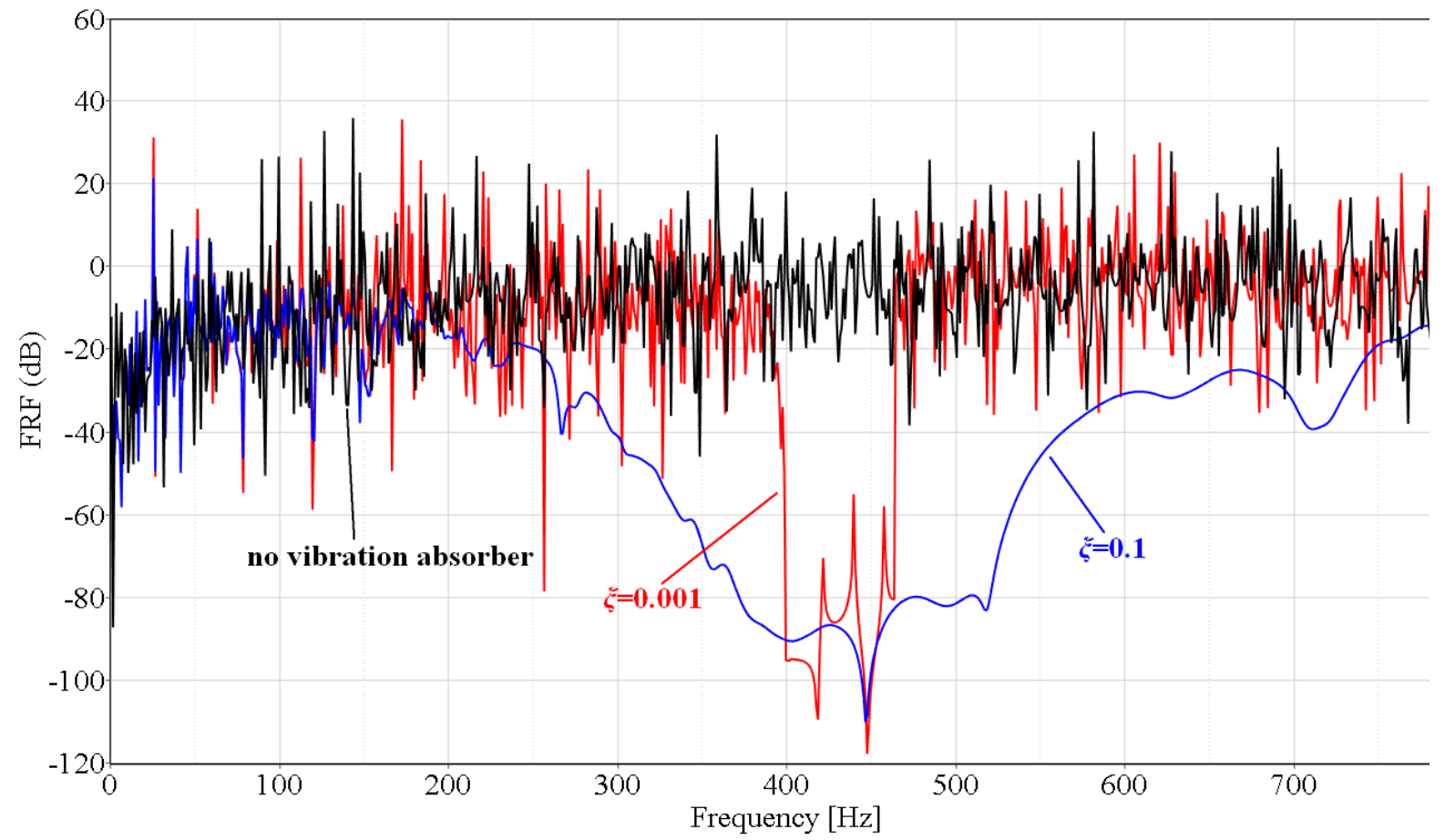

(b)

Fig. 8. Frequency response functions of the laminate acoustic metamaterial in symmetry: $(a)$ response at $x=0.5 L_{\mathrm{a}}$ and $y=0.5 L_{\mathrm{b}}$, and $(b)$ response at $x=0.7 L_{\mathrm{a}}$ and $y=0.5 L_{\mathrm{b}}$ 


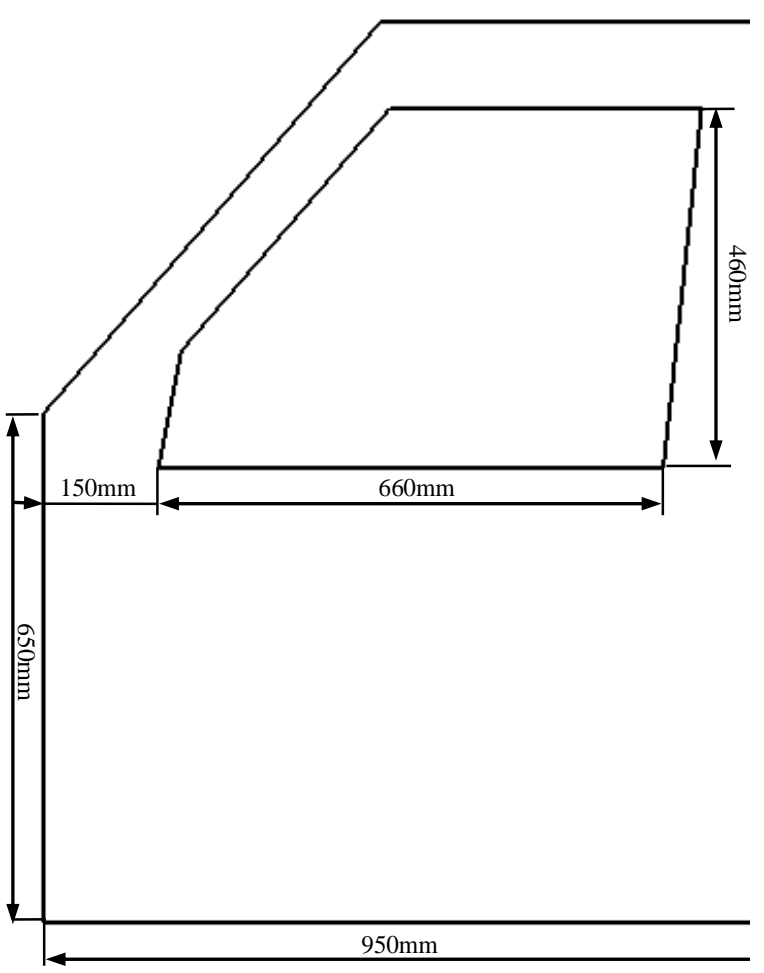

(a)

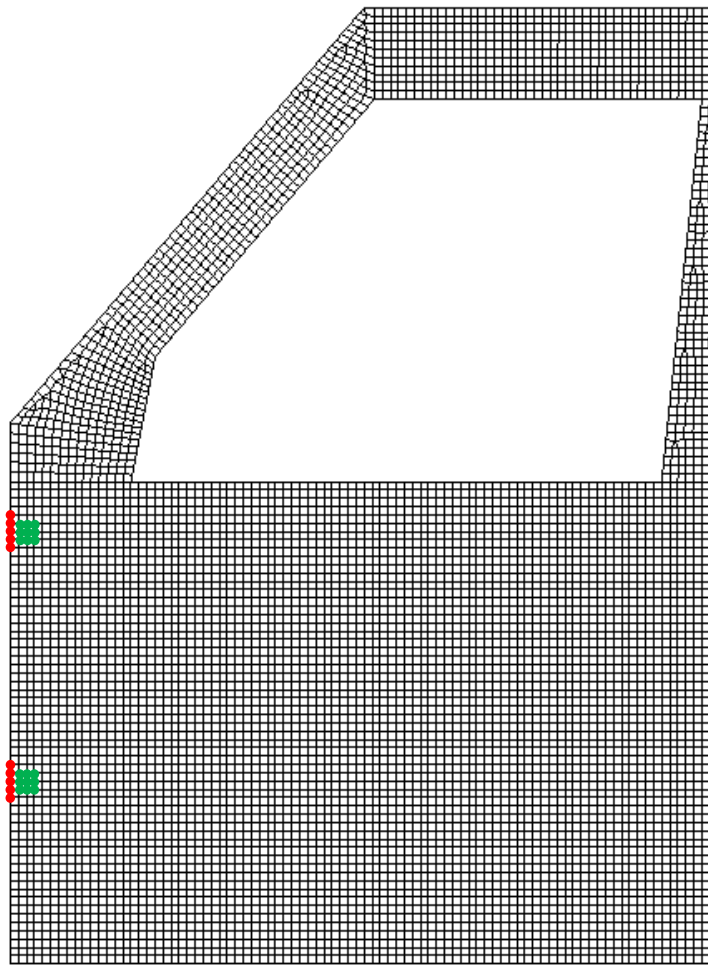

(b)

Fig. 9. A model of vehicle door $(a)$ a 2D model, and $(b)$ a finite model. 


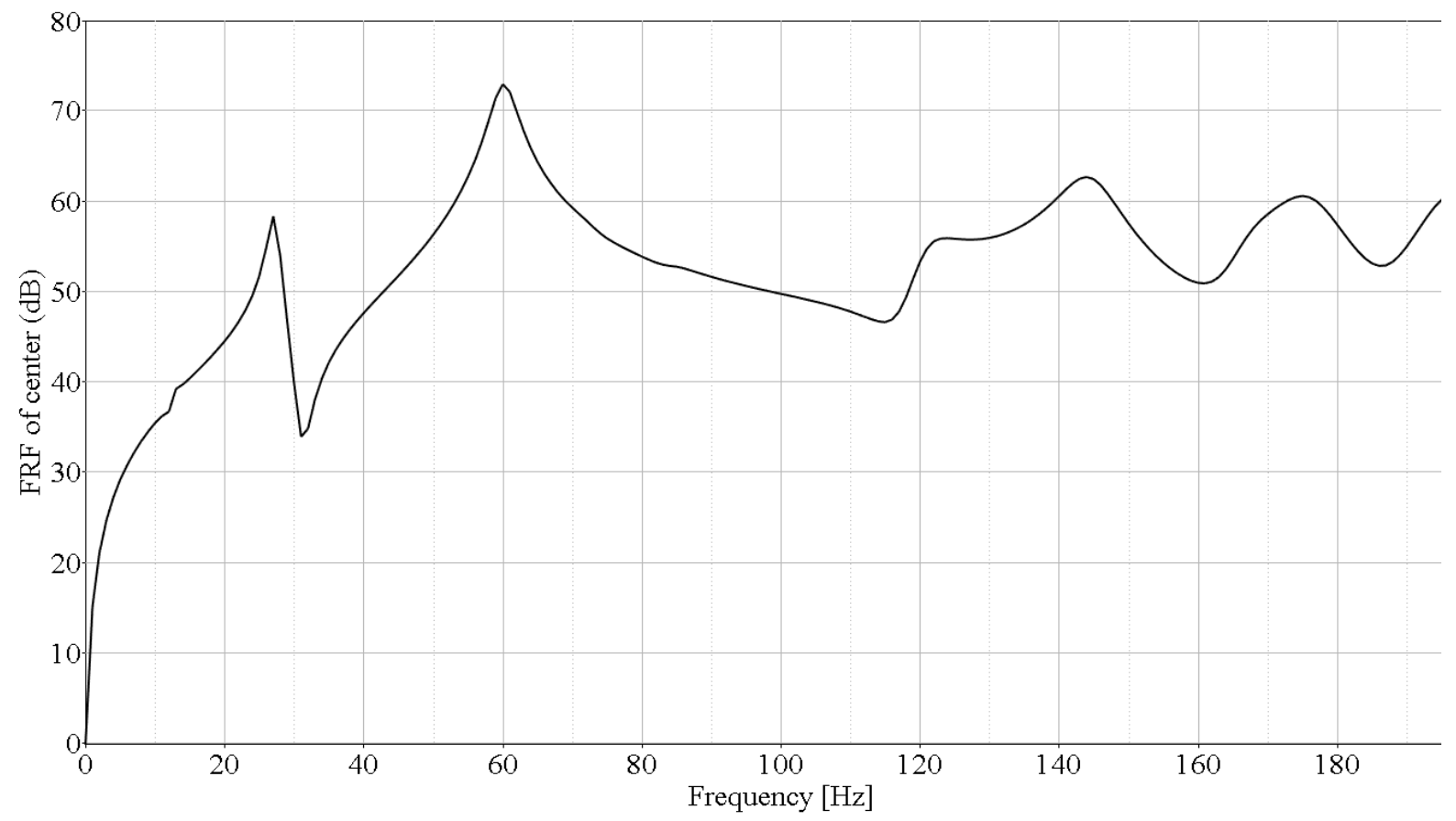

(a)

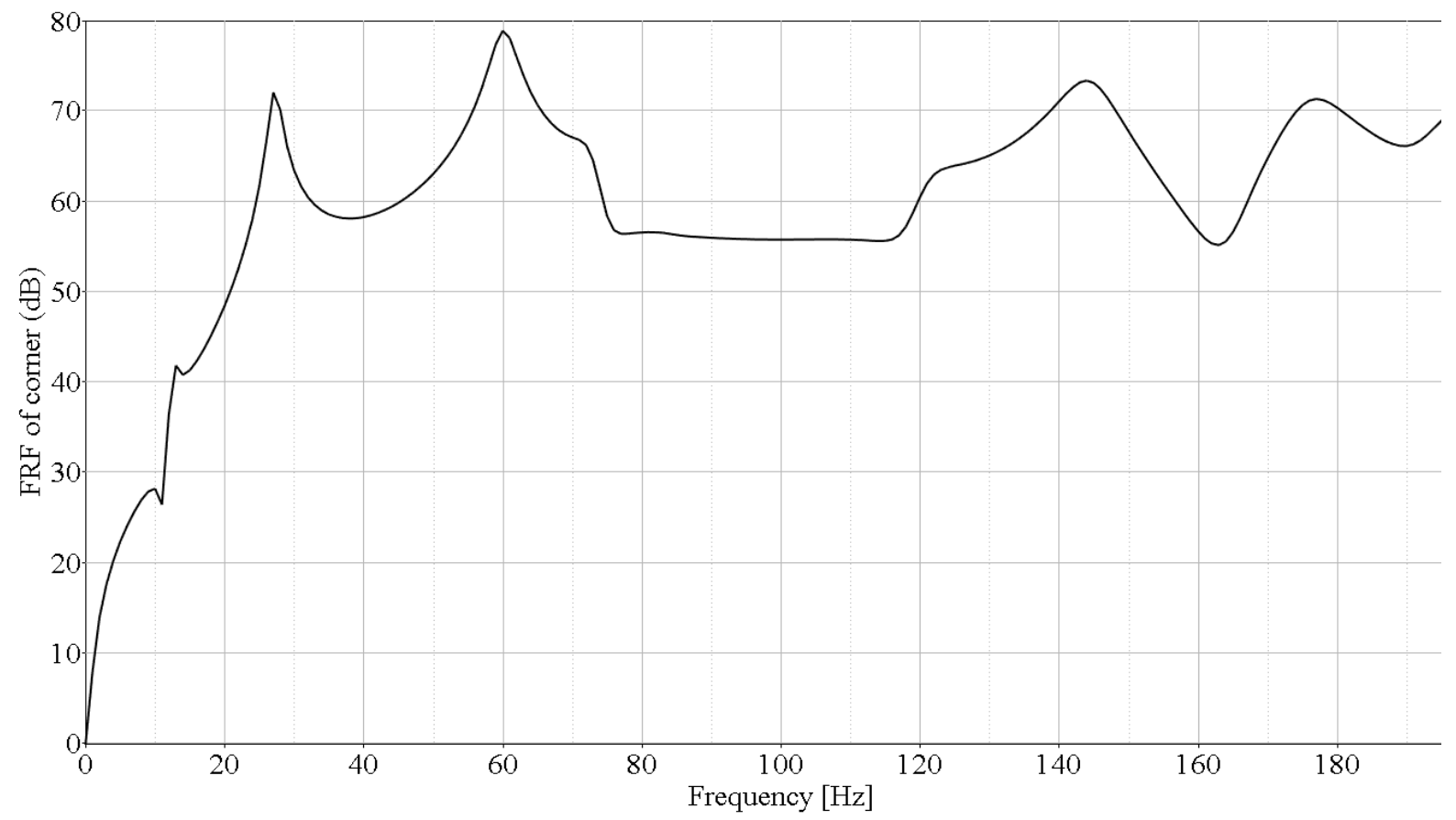

(b)

Fig. 10. FRFs of the vehicle door composed of low-frequency laminate acoustic metamaterials without vibration absorbers: (a) the center node at $x=0.47 \mathrm{~m}$ and $y=0.29 \mathrm{~m}$ and (b) the corner node at $x=0.95 \mathrm{~m}$ and $y=0 \mathrm{~m}$. 


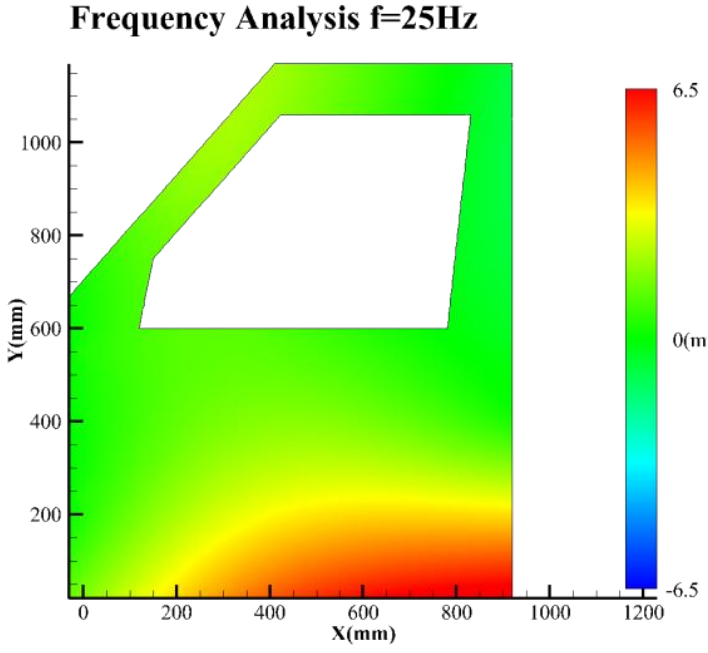

(a)

Frequency Analysis $\mathbf{f}=\mathbf{5 5 H z}$

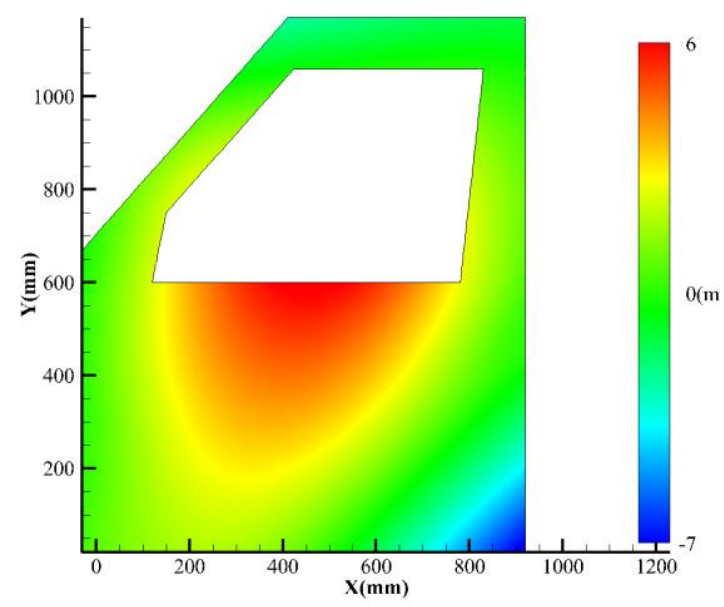

(c)

\section{Frequency Analysis $\mathrm{f}=\mathbf{1 4 5 H z}$}

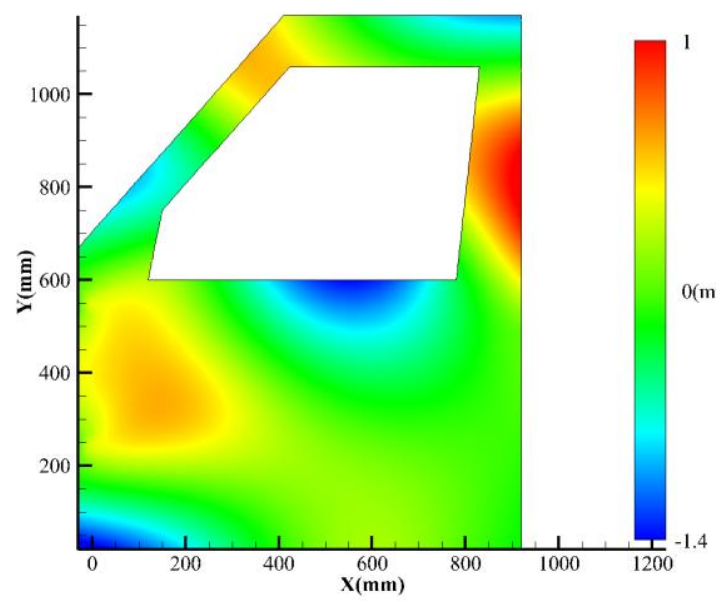

\section{Frequency Analysis $\mathrm{f}=\mathbf{3 0 H z}$}

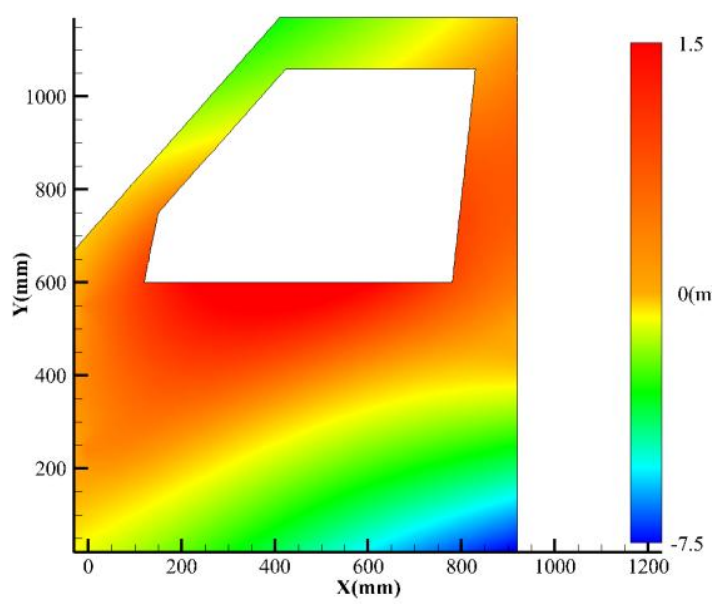

(b)

Frequency Analysis $\mathrm{f}=60 \mathrm{~Hz}$

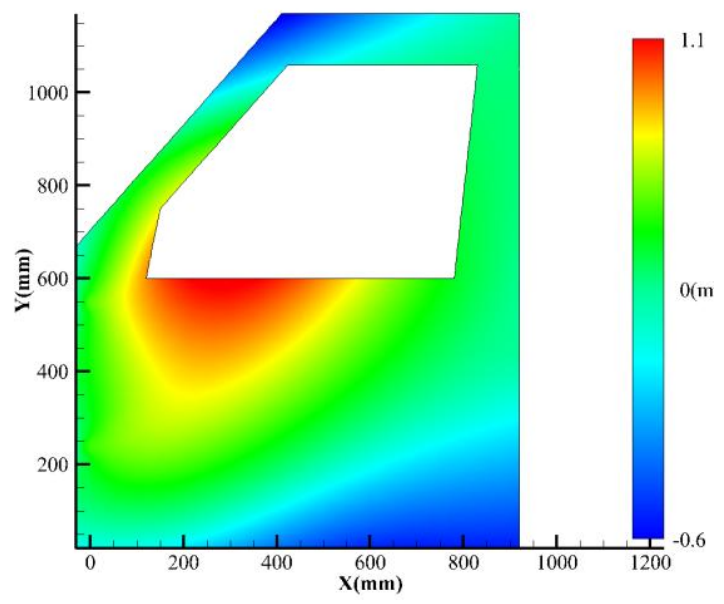

(d)

\section{Frequency Analysis $\mathrm{f}=\mathbf{1 5 0 H z}$}

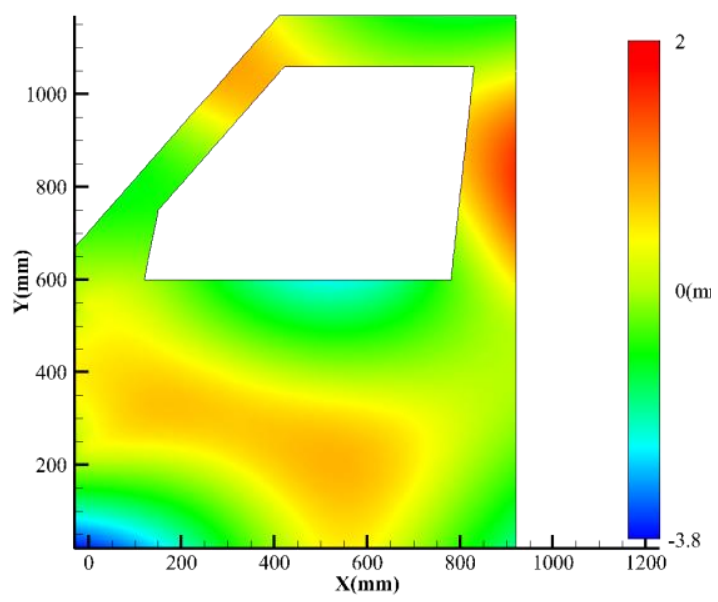


(e)

(f)

Fig. 11. FRA of the vehicle door composed of low-frequency laminate metamaterial without vibration absorbers

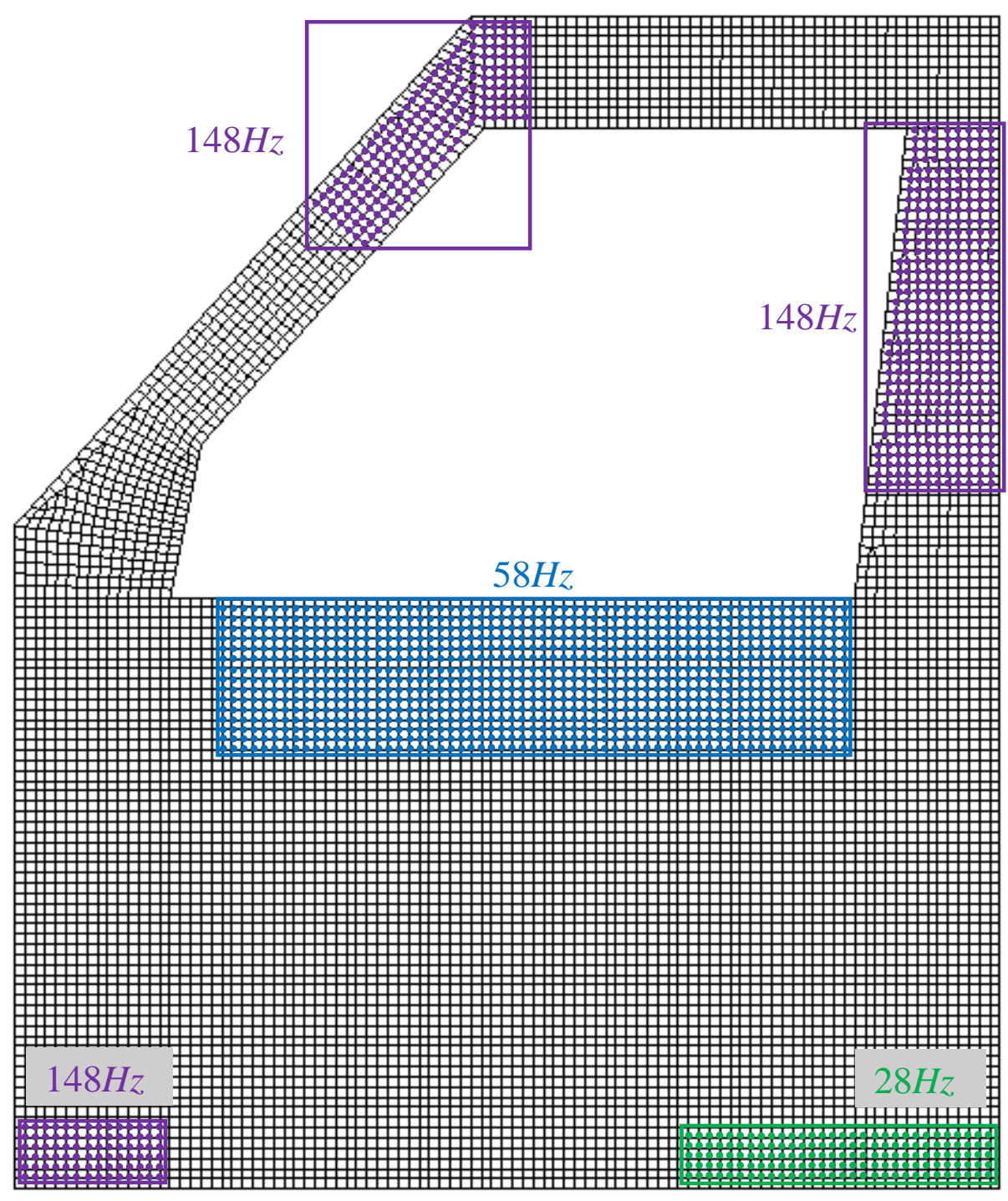

Fig. 12. Distribution of vibration absorbers of different resonant frequencies of the vehicle door composed of low-frequency laminate metamaterial 


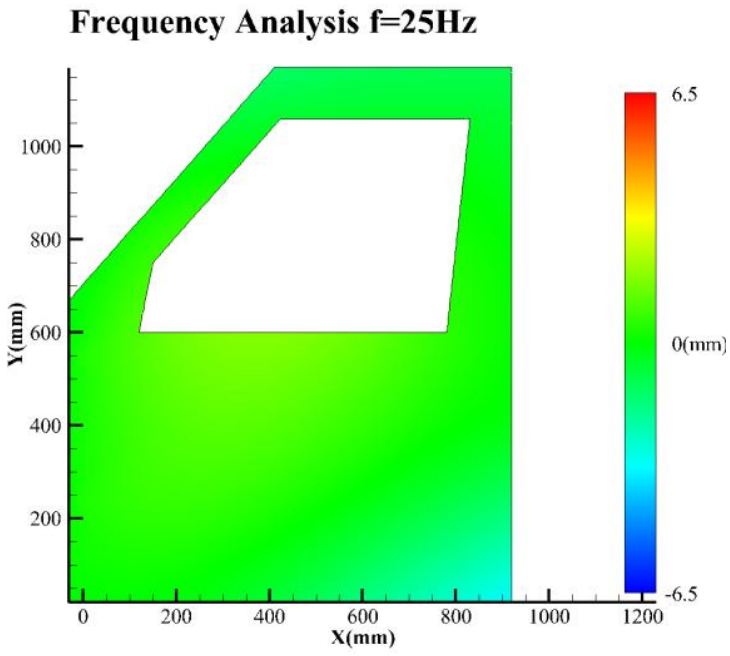

(a)

\section{Frequency Analysis $\mathrm{f}=\mathbf{1 4 5 \mathrm { Hz }}$}

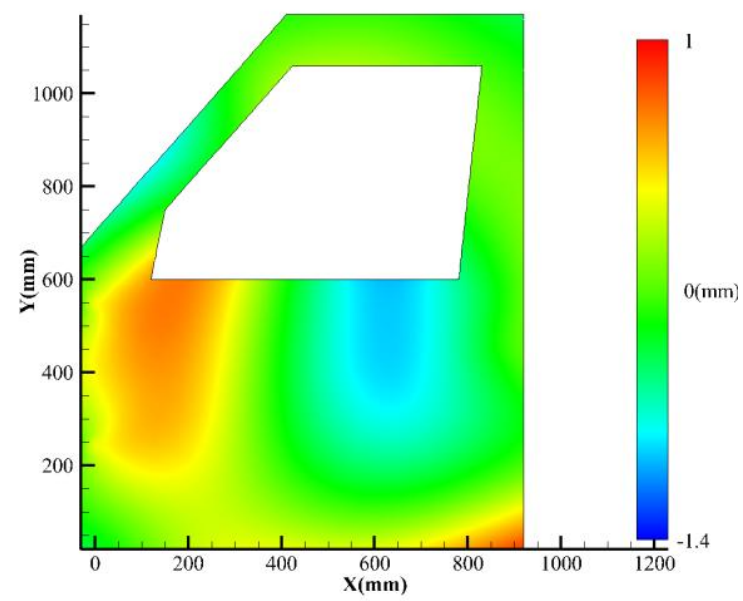

(c)

\section{Frequency Analysis $\mathrm{f}=\mathbf{5 5 H z}$}

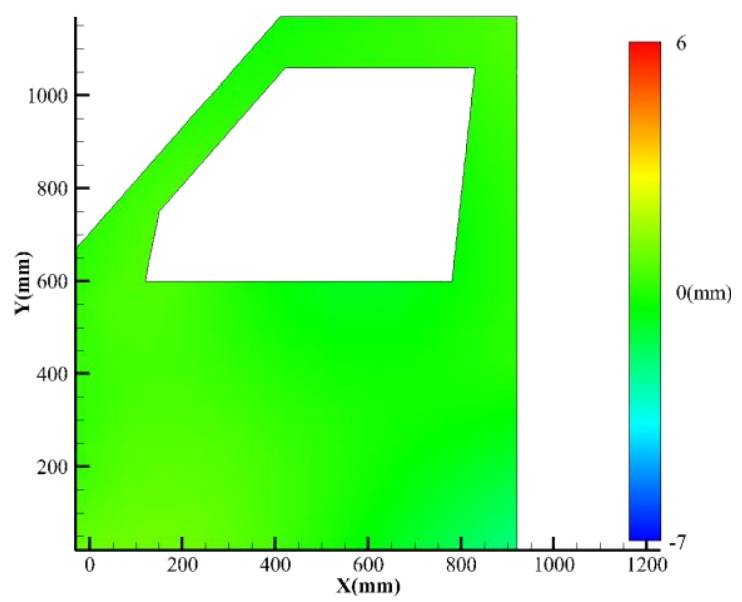

(b)

Frequency Analysis $\mathbf{f}=\mathbf{1 5 0 H z}$

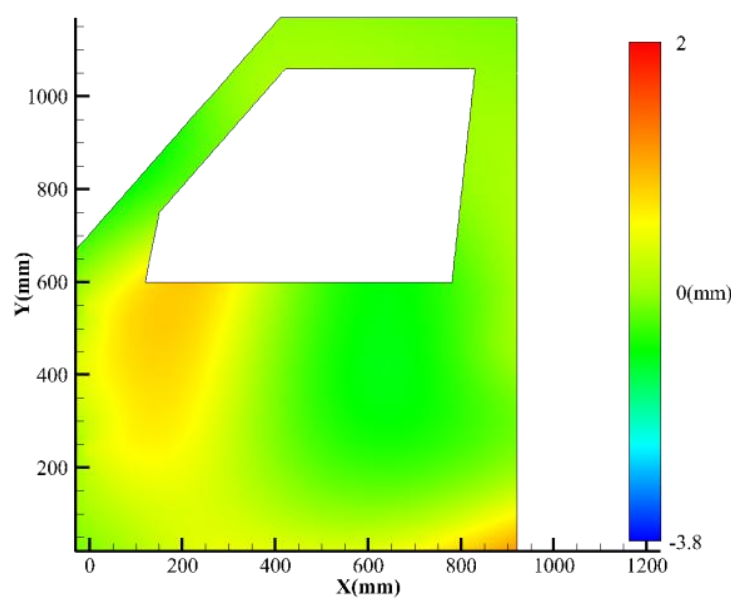

Fig. 13. FRA of the vehicle door composed of low-frequency laminate metamaterial with absorbers

$$
\text { using } \xi=0.001
$$


